\title{
TRIGGERED STAR FORMATION BY MASSIVE STARS
}

\author{
Hsu-Tai Lee ${ }^{1}$ \\ htlee@asiaa.sinica.edu.tw \\ W. P. Chen ${ }^{1,2}$ \\ wchen@astro.ncu.edu.tw
}

\begin{abstract}
We present our diagnosis of the role that massive stars play in the formation of low- and intermediate-mass stars in $\mathrm{OB}$ associations (the $\lambda$ Ori region, the Ori OB1, and Lac OB1 associations). We find that the classical T Tauri stars and Herbig Ae/Be stars tend to line up between luminous $\mathrm{O}$ stars and brightrimmed or comet-shaped clouds; the closer to a cloud the progressively younger they are. Our positional and chronological study lends support to the validity of the radiation-driven implosion mechanism, where the Lyman continuum photons from a luminous $\mathrm{O}$ star create expanding ionization fronts to evaporate and compress nearby clouds into bright-rimmed or comet-shaped clouds. Implosive pressure then causes dense clumps to collapse, prompting the formation of lowmass stars on the cloud surface (i.e., the bright rim) and intermediate-mass stars somewhat deeper into the cloud. These stars are a signpost of current star formation; no young stars are seen leading the ionization fronts further into the cloud. Young stars in bright-rimmed or comet-shaped clouds are likely to have been formed by triggering, which would result in an age spread of several Myrs between the member stars or star groups formed in the sequence.
\end{abstract}

Subject headings: stars: formation — stars: pre-main-sequence — ISM: clouds - ISM: molecules

\section{INTRODUCTION}

Most $\mathrm{O}$ and $\mathrm{B}$ stars are congregated into OB associations (Blaauw 1964) in which young low- (classical T Tauri stars, or CTTSs) and intermediate-mass (Herbig Ae/Be stars,

\footnotetext{
${ }^{1}$ Institute of Astronomy, National Central University, 300 Jungda Road, Jungli 32054, Taiwan

${ }^{2}$ Department of Physics, National Central University, 300 Jungda Road, Jungli 32054, Taiwan
} 
or HAeBe) stellar groups are also found (see the review by Briceño et al. 2006). What is the relationship between the formation of massive stars and that of low-mass stars? Does star formation in an OB association proceed in a bimodal manner for massive and for low-mass stellar groups? If so, which group would form first? It is noted that massive stars have a profound influence on the surrounding molecular clouds. On the one hand, the radiation and energetic wind from a massive star could cause the evaporation of nearby clouds, hence terminating the star formation processes. On the other hand, the massive star could provide "just the touch" needed to prompt the collapse of a molecular cloud which otherwise may not contract and fragment spontaneously. Do massive stars play primarily a destructive or promotional role in star formation in a molecular cloud? Herbig (1962) suggests that low- and intermediate-mass stars form first in an $\mathrm{OB}$ association, but soon after massive O stars appear, the cloud is disrupted, which hinders further star formation. Alternatively, Elmegreen \& Lada (1977) and Lada (1987) propose that low-mass stars form first out of cloud fragments and are distributed throughout the entire molecular cloud. Once the O stars form, their expanding ionization fronts (I-fronts) then play a constructive role in inciting a sequence of star formation in neighboring molecular clouds.

The triggering of star formation by massive stars appears to take place on different length scales (Elmegreen 1998). The Sco OB2 association might be one example of triggered star formation (de Geus et al. 1989). In this case the Upper Centaurus Lupus subgroup was formed first in the middle of the molecular cloud complex, which then prompted star formation on both sides, eventually becoming the Upper Scorpius and Lower Centaurus Crux associations. Preibisch \& Zinnecker (1999, 2006) propose a similar mechanism, but with a series of supernova explosions as the triggering sources. The star formation activities can be sustained as long as stars massive enough are produced in the sequence and there is enough surrounding material. This sequential formation process leads naturally to an age spread among member stars or subgroups (Blaauw 1964), and the stellar aggregates thus formed (out of separate clouds) tend to be sparsely distributed and gravitationally unbound because of the expanding I-fronts or an initially unbound giant molecular cloud (see Clark et al. 2005).

On a smaller scale, the signature of ongoing star formation, such as young stellar jets, evaporated gaseous globules (EGGs) and water masers have been found in the periphery of H II regions (Hester et al. 2004; Hester \& Desch 2005). There are two kinds of triggering mechanisms (e.g., Karr \& Martin 2003), "collect-and-collapse" and radiation-driven implosion (RDI). In the collect-and-collapse scenario first proposed by Elmegreen \& Lada (1977) and recently demonstrated observationally by Deharveng, Zavagno \& Caplan (2005), Zavagno et al. (2006), and Sanchawala et al. (2006), the expanding I-fronts from an H II region piles up a shell of dense gas and dust, in which clumps fragment and collapse to form the next generation of stars. In the RDI scenario (Bertoldi 1989; Bertoldi \& McKee 
1990; Hester \& Desch 2005; Larosa 1983; Kessel-Devnet \& Burkert 2003), the formation sequence begins with photoionization of a nearby molecular cloud by a massive star. The shock fronts embracing the surface of the cloud compress the cloud until reaching the critical density for gravitational collapse resulting in the formation of new stars. The latest star formation, as traced by protostellar cores (Lefloch et al. 2000) or water masers (Healy et al. 2004), takes place at the compressed layer of a cloud. Hester \& Desch (2005) propose a scenario in which an EGG appears when a dense clump is impinged upon by the I-fronts. The photoevaporation then erodes the circumstellar disk into a protoplanetary disk, or a "proplyd" (O'dell et al. 1993). Subsequently formed massive stars can carve out their own cavities to continue the triggering process (Sanchawala et al. 2006). The exposure of the protoplanetary disk in such environments would, in addition to being truncated in size, contain short-lived radio nuclides from the ejecta from one or more nearby supernovae, such as has been observed in meteorites in the Solar System (Hester et al. 2004). Our results substantiate the above sequential star formation scenario by providing clear chronological and positional evidence that massive stars prompt the birth of lower-mass stars out of molecular clouds.

In the Orion star-forming region for example, there is concrete evidence of triggered star formation as manifested by the bright-rimmed clouds (BRCs) in the vicinity of O stars (Lee et al. 2005, hereafter Paper I). These BRCs are considered the remnant of molecular clouds that have been photoionized by a nearby massive star (Sugitani et al. 1991, 1994). According to Paper I, only BRCs that are associated with strong IRAS $100 \mu \mathrm{m}$ emission (tracer of high density) and $\mathrm{H} \alpha$ emission (tracer of the ionization front) show signs of ongoing star formation. Furthermore, CTTSs are more likely seen between the O stars and the BRCs, with those closer to the BRCs being progressively younger, and there are no CTTSs far ahead of the I-fronts.

In this paper we extend the study to the Lac OB1 association as well as include intermediatemass young stars in our sample. We describe in $\S 2$ the archival data and our spectroscopic and imaging observations. In addition to the Lac OB1 sources, some of the stars in Ori OB1 considered in Paper I to be young star candidates have been spectroscopically confirmed. These results are also presented here. Finally, we discuss star-formation activities and histories in $\lambda$ Ori, Ori OB1, and Lac OB1 in $\S 3$, and consider star formation in general in OB associations in $\S 4$. The conclusions are summarized in $\S 5$. 


\section{DATA AND OBSERVATIONS}

\subsection{ARCHIVE DATA}

CTTSs are young stellar objects characterized by their infrared excess. Usually CTTSs are more likely to be spatially closer to a star-forming region than are the weak-line $\mathrm{T}$ Tauri stars (WTTSs). The latter are also PMS stars, but are more evolved than CTTSs in terms of clearing of their inner circumstellar disks. Thus the CTTSs trace more recent star formation. In Paper I, we proposed an empirical set of criteria to select CTTS candidates from the 2MASS Point Source Catalog (Cutri et al. 2003). In this paper, we apply the same selection procedure (e.g., 2MASS colors, good photometric qualities, and exclusion of extended sources) but include young intermediate-mass stars, the HAeBe stars into our sample. Different young stellar populations, WTTSs, CTTSs, and HAeBe stars occupy distinctly different regions in the 2MASS color-color diagram (see paper I). The HAeBe stars in general exhibit larger infrared excess than CTTSs do. Therefore we select as HAeBe star candidates $2 \mathrm{MASS}$ point sources with colors redder than the line defined by $\left(m_{J}-m_{H}\right)-$ $1.7\left(m_{H}-m_{K}\right)+0.450=0$; CTTS candidates are selected by the same method described in paper I, namely between the two parallel lines, $\left(m_{J}-m_{H}\right)-1.7\left(m_{H}-m_{K}\right)+0.0976=0$ and $\left(m_{J}-m_{H}\right)-1.7\left(m_{H}-m_{K}\right)+0.450=0$, and above the dereddened CTTS locus (Mever et al. 1997), $\left(m_{J}-m_{H}\right)-0.493\left(m_{H}-m_{K}\right)-0.439=0$.

Table 1 shows the fields in the $\lambda$ Ori region, OriOB1, and Lac OB1 studied in this paper, which include 7 BRCs, one comet-shaped cloud and two control regions. In addition to the 2MASS point-source database from which we select our CTTS and HAeBe candidates, we also make use of the $\mathrm{H} \alpha$ emission survey data (Finkbeiner 2003; Gaustad et al. 2001; Dennison et al. 1998; Haffner et al. 2003), $E(B-V)$ reddening (Schlegel et al. 1998), IRAS $100 \mu \mathrm{m}$, and CO (Dame et al. 2001) emission to trace, respectively, the distribution of the ionization fronts, cloud extinction, IR radiation, and molecular clouds with respect to the spatial distribution of our young star sample.

\subsection{SPECTROSCOPIC OBSERVATIONS}

The spectra of bright CTTS and HAeBe candidates were taken at the Beijing Astronomical Observatory (BAO) and at the Kitt Peak National Observatory (KPNO). At the BAO, low-dispersion spectra with a dispersion of $200 \AA \mathrm{mm}^{-1}$, corresponding to $4.8 \AA$ pixel $^{-1}$, were taken with the $2.16 \mathrm{~m}$ optical telescope from 2003 October 31 to November 3, and on 2004 September 5-6. An OMR (Optomechanics Research, Inc.) spectrograph was used with a Tektronix 1024×1024 CCD detector covering 4000-9000 A. These spectra were used to con- 
firm the young stellar nature (e.g., the $\mathrm{H} \alpha$ and other characteristic emission lines) of the PMS star candidates selected on the basis of the 2MASS colors.

Medium-dispersion spectra for a selected set of sample stars were taken with the KPNO $2.1 \mathrm{~m}$ telescope on 2004 January $2-5$. The GoldCamera spectrometer, with a Ford $3 \mathrm{~K} \times 1 \mathrm{~K}$ CCD with $15 \mu \mathrm{m}$ pixels, was used with the grating \#26new, giving a dispersion of $1.24 \AA$ pixel $^{-1}$. These medium-dispersion spectra allowed us to identify the lithium absorption at $6708 \AA$, the spectral signature of a low-mass PMS star.

All the spectroscopic data were processed with the standard NOAO/IRAF packages. After correction for bias and flat-fields, the IRAF package KPNOSLIT was used to extract and to calibrate the wavelength and flux of each spectrum. To check the legitimacy of our selection criteria, we also observed two control fields, in addition to the star-forming clouds. All the fields included in this study are summarized in Table 1.

\subsection{IMAGING OBSERVATION}

The BRCs were imaged on 2004 November 3-8 using the $1 \mathrm{~m}$ telescope at the Lulin Observatory in Taiwan (Table 2). A PI1300B (Roper Scientific) CCD camera was used, which has $1340 \times 1300$ pixels, each $20 \mu \mathrm{m}$ square, yielding a $\sim 11^{\prime}$ field of view. $\mathrm{H} \alpha$ $\left(\lambda_{c}=6563 \AA, \Delta \lambda(\mathrm{FWHM})=30 \AA\right)$ images were taken for all BRCs. In addition, LBN 437

was observed with an [S II] $\left(\lambda_{c}=6724 \AA, \Delta \lambda(\mathrm{FWHM})=80 \AA\right)$ filter. For every target field tens of images were taken, each with an exposure time of 120 to $300 \mathrm{~s}$. The images were processed for bias, dark and flat-fielding corrections with the standard procedures.

\subsection{OBSERVATIONAL RESULTS}

The main purpose of the spectral observations was to identify PMS star candidates and to validate the selection criteria for HAeBe stars. The imaging observations can help us to trace the distribution of the I-fronts in the BRCs. By combining the spectral and imaging observations, we can study the spatial distribution of PMS stars relative to I-fronts in BRCs.

Tables 3, 4, and 5 list, respectively, the CTTSs (plus some CTTS candidates), HAeBe stars, and non-PMS sources identified from spectroscopic observations. In Table 3, stars 131 CTTSs are in Orion and 32-40 CTTSs are in Lacerta. In Table 4, stars 41-48 are HAeBe stars in the Orion region, whereas the others are in the Lacerta region. We derive the $\mathrm{H} \alpha$, $[\mathrm{O} \mathrm{I}]$, and $[\mathrm{S} \mathrm{II}]$ equivalent widths of the CTTSs. Some of the CTTSs listed in Table 3 do not show lithium absorption, but exhibit other CTTS characteristics, such as the $\mathrm{H} \alpha$, Ca II, 
and/or forbidden [O I] and [S II] emission line(s) in their spectra. Since most of these spectra show veiling, their Li absorption line might be veiled by continuum radiation. Thus they are included in the CTTS sample (Table 3) even though the Li line is not readily discernible. CTTSs without a Li absorption line are not unusual; recently White \& Hillenbrand (2005) also found a lithium-depleted CTTS, St 34, in the Taurus-Auriga T association. Figure 1 presents an example of the spectra of a CTTS and a HAeBe star. No PMS stars were found in any of the two control fields; most of the sources there are either carbon stars or M giants.

Figure 2 and Figure 3 show, respectively, the Trapezium and the $\lambda$ Ori regions in Orion, with the CTTSs (stars 1-31 in Table 3) and HAeBe stars (stars 41-48 in Table 4) being marked. The boxes mark the fields of the $\mathrm{H} \alpha$ images presented in Fig. 4. It is clear that the BRCs are outlined by the $\mathrm{H} \alpha$ emission, and that some PMS stars are spatially close to the I-fronts.

Figure 5 displays the IRAS $100 \mu \mathrm{m}, \mathrm{H} \alpha$ and CO emission maps of the Lac OB1 association. The PMS stars in Table 3 and Table 4 are again marked. The box indicates the LBN 437 region shown in Figure 7. LBN 437 is a comet-shaped BRC (Olano et al. 1994). The HAeBe star V375 Lac (star 52 in Table 4) associated with this cloud is believed to be the exciting source of the parsec-scale Herbig-Haro outflow HH 398 (McGroarty et al. 2004).

In Paper I it was shown that CTTSs exhibiting continuous or veiled spectra with [O I] and/or [S II] forbidden lines, originating from jets or winds seen commonly in Class I sources (Kenyon et al. 1998), tend to be redder, which is suggestive of a younger age, than those without. A color-color diagram of the PMS stars in Tables 3 and 4 is plotted as Fig. 6, the results agree with our previous work. This correlation extends to HAeBe stars, in that HAeBe stars with forbidden line(s) are mostly located on the upper right of the 2MASS color-color diagram. As an alternative to being younger, a CTTS with forbidden lines could be the result of reduced photoevaporation of the circumstellar disk, e.g., by being away from a luminous star or shielded by a molecular cloud. Only 14 of the 40 CTTSs and 4 of the 13 HAeBe stars in our sample show forbidden line(s) in their spectra. In other words, about one third of the PMS stars with strong infrared excess exhibit forbidden line(s). Typical CTTS ages are a few Myr (Kenyon \& Hartmann 1995), with those with forbidden lines representing an even younger sample, probably no more than a couple Myr old. The [S II] line is only present in Star 2, a CTTS with a strong infrared excess and strong [O I] (equivalent width > $10.5 \AA$ ). In our sample of CTTSs there is no correlation between the $\mathrm{H} \alpha$ equivalent widths and the presence of forbidden lines, or between the $\mathrm{H} \alpha$ equivalent widths and the 2MASS colors.

The success rate of spectroscopic confirmation of CTTS and HAeBe candidates is extremely high for $\lambda$ Ori, Ori OB1 or Lac OB1. Candidates closely associated with star-forming 
regions all turned out to be bona fide young stars with essentially no exception, whereas the regions away from molecular clouds are mostly populated by evolved stars (e.g., carbon stars or M giants). The 2MASS database enables us to effectively trace recent star formation on a large scale, without any a priori bias toward prominent H II or reflection nebulae which are obvious targets to search for young stellar objects. For example, stars 35-38 in our sample are confirmed to be young stars. They are located away from prominent nebulosity, so it might otherwise be difficult to recognize them as young stars in a targeted survey.

\section{STAR FORMATION IN THE ORI OB1 AND LAC OB1 ASSOCIATIONS}

Star formation triggered by the RDI mechanism has several characteristics which can be diagnosed observationally: (1) The remnant cloud is extended toward, or pointing to, the massive stars. (2) The young stellar groupings in the region are roughly lined up between the remnant clouds and the luminous star. (3) Stars closer to the cloud, which have formed later in the sequence, are younger in age, with the youngest stars being in the interacting region, i.e., along the bright rim of the cloud. (4) No young stars exist far behind the BRC. In particular, (3) and (4) are in distinct contrast to the case of spontaneous star formation, which conceivably would not have left such distinguishing temporal and positional signposts. In Table 6 we summarize the different outcomes of the triggered versus spontaneous star formation processes.

In Paper I, we presented evidence supporting the induced star formation in six Orion BRCs, namely B 30, B 35, Ori East, IC 2118, LDN 1616, and LDN 1634. Here, we present further spectroscopic observations of the Orion sources, classified as young star "candidates" in Paper I and extend our sample to include the Lac OB1 region. Combined with the earlier Ori OB1 results, this reinforces the links between massive stars, BRCs, and the formation of low-mass stars. Furthermore, our young star sample now contains not only CTTSs, but also young intermediate-mass stars, rendering a more comprehensive understanding of the origin of stellar masses in an $\mathrm{OB}$ association.

\subsection{STAR-FORMING ACTIVITIES IN THE ORION REGION}

\subsubsection{IC 2118, LDN 1616, LDN 1634, AND ORIEAST}

IC 2118, LDN 1616, and LDN 1634 are three isolated BRCs around the Trapezium to the west of the Orion A. Another BRC, Ori East, can be found to the north-east of the Trapezium. All these BRCs point roughly to the Trapezium (Fig. 2), indicative of the Trapezium and/or 
the Orion-Eridanus superbubble being the shaping source of these BRCs (Alcalá et al. 2004; Stanke et al. 2002; Kun et al. 2001, 2004).

In this region most CTTSs with forbidden line(s), i.e., those of younger ages, are spatially close to the BRCs, e.g., stars 1, 2, and 30 in relation to LDN 1616, IC 2118, and Ori East, respectively. Star 7 is also associated with a remnant molecular cloud (Ogura \& Sugitani 1998) (their cloud 6).

\subsection{2. $B 30$ AND $B 35$}

B 30 and B 35 are two BRCs associated with an H II region excited by the O8 III star $\lambda$ Ori and surrounded by a ring-shaped molecular cloud (Lang et al. 2000). Duerr et al. (1982) find some $80 \mathrm{H} \alpha$ stars in the $\lambda$ Ori region, most of which are distributed as a barlike structure extending from either side of $\lambda$ Ori to B 30 and to B 35. Dolan \& Mathieu (1999, 2001, 2002) present photometric and spectroscopic studies of the young stellar population

in the $\lambda$ Ori region. They suggested the ring-shaped molecular cloud to be caused by a supernova explosion that terminated recent star formation in the vicinity.

It is likely that $\lambda$ Ori is the triggering source responsible for the star formation in B 30

and B 35. It is found that photoevaporative flows (Hester et al. 1996) stream out of the surfaces of them; this is a demonstration of the interaction between a massive star and a molecular cloud (Fig. 3). Here again we see that stars with forbidden lines, i.e., stars 20, 22, and 44 in relation to B 30, and stars 25 and 26 to B 35, are all physically close to a BRC.

\subsection{STAR FORMATION HISTORY IN ORI OB1 AND THE $\lambda$ ORI REGION}

It is suggested that star formation is triggered by the $\mathrm{O}$ stars and/or by the superbubbles in LDN 1616 (Alcalá et al. 2004; Stanke et al. 2002) and in IC 2118 (Kun et al. 2001, 2004). All BRCs in OriOB1 which show evidence of star formation being triggered by nearby massive stars are found associated with strong IRAS $100 \mu \mathrm{m}$ and $\mathrm{H} \alpha$ emission (Fig. 4). In every case a sequential process - that PMS stars closer to the triggering stars are older than those closer to the BRCs - can be clearly witnessed (Paper I).

The same phenomena are also seen near $\lambda$ Ori. Initially, the B 30 and B 35 clouds might have extended toward $\lambda$ Ori, perhaps forming a barlike structure. The I-fronts from $\lambda$ Ori then propagated through the clouds, prompting star formation on both sides, thereby resulting in the lining up of the PMS stars, in an age sequence, between $\lambda$ Ori and the B 30 
and B 35 clouds.

In Paper I we show that the CTTSs that are spatially close to BRCs are among the brightest, just revealing themselves on the birthline and beginning to descend down the Hayashi tracks. We also find no young stars far behind the I-fronts, i.e., embedded in the BRCs. These photoevaporated clouds typically have low extinction so that any PMS stars cannot have escaped the 2MASS detection. In both Ori OB1 and the $\lambda$ Ori region therefore, we see the predomination of triggered star formation, as evinced in the cloud morphology, star grouping orientation, and star formation sequence.

\subsection{STAR FORMATION ACTIVITIES IN LAC OB1}

The Lac OB1 association, at a distance of $\sim 360$ pc (de Zeeuw et al. 1999), is one of the nearest OB associations. Blaauw (1958) divides Lac OB1 into 2 subgroups, "a" and "b", on the basis of stellar proper motions and radial velocities. The entire Lac OB1 covers the region of the sky from $90^{\circ}<\ell<110^{\circ}$ and $-5^{\circ}<b<-25^{\circ}$ (de Zeeuw et al. 1999). Lac OB1b occupies an area with a radius of $\sim 5^{\circ}$ centered around $(\ell, b)=\left(97^{\circ} .0,-15^{\circ} .5\right)$ and Lac OB1a occupies the remaining area. The Lac OB1b harbors the only O star (O9 V), $10 \mathrm{Lac}$, in the Lac OB1 association. Our study discusses two regions in Lac OB1 known to have current star-forming activities, LBN 437 and GAL 110-13, a BRC and a comet-shaped cloud, respectively.

\subsection{1. $L B N 437$}

LBN 437 is at the edge of an elongated molecular cloud (Olano et al. 1994) and on the border of the H II region S126 excited by the nearby O star, 10 Lac. Hereafter we call this elongated molecular cloud the "Lac molecular cloud" (Fig. 5). Between 10 Lac and LBN 437 there is a small stellar group (Fig. 8) which includes 5 CTTSs (stars 35-38 in Table 3, plus the CTTS candidate 2MASS J22354224+3959566, for which we do not have spectroscopic observations) and one HAeBe star (star 53 in Table 4). The HAeBe star is an IRAS source, IRAS $22343+3944$. We can identify IRAS $22343+3944$ as the counterpart of star 53, because this star shows a near-infrared excess and is located within the positional error for IRAS $22343+3944$. Hereafter we refer to this 6 -star system as the IRAS $22343+3944$ group (Fig. 5). The size of the IRAS $22343+3944$ group is about $24^{\prime}$, which corresponds to $\sim 2.5$ pc at 360 pc. 


\subsubsection{GAL $110-13$}

GAL 110-13 is an isolated and elongated molecular cloud (Fig. 9) at a distance of $\sim 440$ pc (Odenwald et al. 1992). Its head-tail, comet-like shape suggests compression by ram-pressure, perhaps as a result of a recent cloud collision (Odenwald et al. 1992). Star formation takes place on the compressed side of GAL 110-13, e.g., the location of the CTTS star 40 (BM And) and the nebula vdB 158 reflecting light from the B9.5V star HD 222142 (Magakian 2003). In addition to HD 222142 there are two other late B-type stars in the vicinity, HD 222046 and HD 222086. All three B stars and star 40 share common proper motions (Zacharias et al. 2004, data extracted from the Second U.S. Naval Observatory CCD Astrograph Catalog), which are consistent with those of the Lac OB1 groups (ESA 1997), as summarized in Table 7. GAL 110-13 is located near the border of the Lac OB1 association, at a distance not very different from that of Lac OB1. GAL 110-13 was not included as part of Lac OB1 by de Zeeuw et al. (1999), but our analysis suggests that the cloud, together with the young stars associated with it, is likely part of Lac OB1a.

GAL 110-13 is elongated and roughly points toward 10 Lac (see Fig. 55). This implies that Lac OB1b or 10 Lac alone is responsible for shaping the cloud. Either shock fronts from a supernova or ionization fronts from a massive star could have caused the shape of this cloud as well as the spatial distribution of young stars in GAL 110-13. In the supernova scenario a star in Lac OB1b more massive than 10 Lac exploded, and, assuming that Lac OB1b and $10 \mathrm{Lac}$ are at the same distance from us (i.e., $358 \mathrm{pc}$ ), it would take a few hundred thousand years for the supernova shock waves (at a speed of hundreds of $\mathrm{km} \mathrm{s}^{-1}$ ) to propagate across the $126 \mathrm{pc}$ separation to arrive, compress, and finally prompt the formation of stars within GAL 110-13. Additional evidence in support of this supernova scenario comes from the B5V star, HD 201910, which is supposed to be a runaway star kicked out from a binary system in Lac OB1b, when one of the component stars became a supernova (Blaauw 1961; Gies \& Bolton 1986). If this is so, the kinematic time scale of the star, $2.7 \mathrm{Myr}$, suggests that a supernova explosion occurred some $2.7 \mathrm{Myr}$ ago and the associated shocks subsequently caused GAL 110-13 to develop its present cometary shape.

An alternative explanation is due to compression by ionization fronts from a massive star, which would be a less destructive method for star formation than a supernova explosion (Lefloch et al. 2002). We propose a scenario in which $10 \mathrm{Lac}$ - still in existence now-was born at the edge of the Lac molecular cloud, similar to that presented in Figure 5 , but with the cloud originally being more extended toward 10 Lac. Soon after its birth, 10 Lac ionized the surrounding molecular clouds, exposing itself to the intercloud medium. Assuming that most of the UV photons of 10 Lac shortward of the Lyman limit were used to ionize the intercloud medium, then given a typical intercloud material density $\sim 0.2 \mathrm{~cm}^{-3}$ (Spitzer 
1998; Dyson \& Williams 1997), the I-fronts would travel the 126 pc distance from 10 Lac to GAL 110-13 in about $2 \mathrm{Myr}$, a time scale still shorter than the main sequence life time of $\sim 3.6$ Myr of 10 Lac (Schaerer \& de Koter 1997). Regardless of which scenario actually happened, a supernova shock front or an ionization front, Lac OB1b is likely responsible for the creation of GAL 110-13 and the associated stellar group.

\subsection{STAR FORMATION HISTORY IN LAC OB1}

In LBN 437, star 52 (V375 Lac) is the only young star located at the edge of the Lac molecular cloud, and interestingly there is no CTTS or HAeBe candidate behind the interaction region. To check whether any PMS stars could have escaped the 2MASS detection limit of $J=15 \mathrm{mag}$ as a result of excessive dust extinction, we created the $E(B-V)$ map of the Lac molecular cloud (Schlegel et al. 1998) (as done for the BRCs in Ori OB1; see Paper I). It is assumed that the $J$-band luminosities of the embedded PMS stars are the same as those of the visible PMS stars outside the cloud, namely the IRAS 22343+3944 group. Like other BRCs we have analyzed the overall extinction in the Lac molecular cloud is low, and the probability of nondetection is 0.014 . This means that there are indeed no embedded PMS stars and hence no ongoing star formation in the Lac molecular cloud.

The elongated Lac molecular cloud associated with LBN 437 (Fig. 5) may be just the remnant of a molecular cloud, which was originally perhaps larger, extending as far as to 10 Lac. Upon the birth of 10 Lac, its energetic photons evaporated and compressed the cloud, shaping the cloud into a pillar, similar to the case of GAL 110-13. The IRAS 22343+3944 group and star 52 were then born on the compressed side of the cloud. At least 3 stars in the IRAS $22343+3944$ group exhibit forbidden lines, which is suggestive of their youth. Star 52 is likely even younger because it is the exciting source of an HH outflow. Apparently star 52 is the latest product in the star formation sequence by 10 Lac in this cloud.

Odenwald et al. (1992) derived a 30\% star formation efficiency for GAL 110-13. This is much higher than that of the few percent typical in star-forming regions (White et al. 1995). Extinction is low in GAL 110-13, with an $\mathrm{A}_{J}$ less than $0.48 \mathrm{mag}$, as estimated from its $E(B-V)$ values, so the cloud is insufficiently dense to hide from our detection any embedded young stars similar to star 40. As in the case for Ori OB1 (Paper I), the BRCs in Lac OB1 also tend to have a relatively low dust extinction. Such a low density condition is unfavorable for spontaneous, global cloud collapse. Star formation however could take place at the interaction layer (the bright rim) of a molecular cloud. A stellar group could form, such as witnessed in the IRAS 22343+3944 and GAL 110-13 groups. 
Blaauw (1958) and Blaauw (1964, 1991) derived the ages of Lac OB1a and Lac OB1b, on the basis of stellar proper motions and radial velocities: 16-25 Myr and 12-16 Myr, respectively. Both these ages are too old to be consistent with the existence of 10 Lac (with a lifetime of less than $\sim 3.6 \mathrm{Myr}$ ) and the CTTSs (typically aged a few Myr) in the region. Thus Lac OB1a and Lac OB1b could not have formed at the same place and at the same time, because with a typical velocity dispersion of a few kilometers per second for an OB association (de Zeeuw et al. 1999; de Bruijne 1999), the two subgroups could not traverse the distance of 30-80 pc now between them. We propose that both Lac OB1a and Lac OB1b are no more than a few Myr old, and Lac OB1a is younger than Lac OB1b. Figure10 shows the color-magnitude diagrams reconstructed from de Zeeuw et al. (1999) for the two subgroups. It can be seen that the stars in Lac OB1b form a clear main sequence, whereas those in the subgroup Lac OB1a are widely scattered to the right of the sequence. Some stars in Lac OB1a may well still be in the PMS phase, hence we postulate a younger age for Lac OB1a than for Lac OB1b.

It is possible that Lac OB1b was formed first after which the expanding I-fronts from Lac OB1b triggered new generations of stars along the Lac molecular cloud, the IRAS 22343+3944 group and star 52. A subsequent supernova or I-front then initiated the formation of stars in Lac OB1a; eventually the "birth wave" reached GAL 110-13.

\section{STAR FORMATION IN OB ASSOCIATIONS}

Triggered star formation has been suggested to have occurred close to $\mathrm{H}$ II regions (Hester et al. 2004; Hester \& Desch 2005). Our study finds clear chronological and positional causality of such processes on larger scales. In $\lambda$ Ori, Ori OB1 and Lac OB1, we see supporting evidence of triggered star formation. The UV photons from an O star create expanding I-fronts which evaporate and compress nearby molecular clouds, thereby shaping the clouds into BRCs or comet-shaped clouds. The next generation of stars can then form efficiently, perhaps in groups, out of the compressed material. The resulting newly formed stars would line up between the massive star and the molecular coulds in a formation and hence age sequence. Stars at least as massive as late Herbig Be types could be formed via this process (see Table 4). These stars would reach the main sequence with even earlier spectral types. Triggered star formation could therefore produce not only low-mass stars, but also intermediate-mass or even massive stars. In our sample, the HAeBe stars and CTTSs seem to be distributed spatially differently relative to a BRC, in the sense that the CTTSs tend to be located near the surface of a BRC, whereas the HAeBe stars appear preferentially to reside deeper into a BRC(e.g., star 44 in B 30, star 52 in LBN 437, and star 41 in LDN 1616). 
What we see in $\lambda$ Ori, Ori OB1, and Lac OB1 is in contrast to the scenario proposed by Elmegreen \& Lada (1977) and Lada (1987) for which massive stars are formed in shocked cloud layers by triggering, whereas low-mass stars are formed spontaneously via cloud collapse and fragmentation. A global cloud collapse would lead to starbirth spreading throughout the cloud, but this was not observed in our study. Instead, no young stars are found embedded in clouds far behind the I-fronts. More than mere "fossil" molecular clouds, the BRCs present convenient snapshots of how star formation must have proceeded in an OB association. When prompted to form, massive stars appear to favor denser environments where photoevaporation is relatively weak. In comparison, when a dense molecular core near the ionization layer (i.e., current cloud surface) collapses, the accretion process has to compete with the mass loss arising from photoevaporation, leading to the formation of less massive stars or even substellar objects (Whitworth \& Zinnecker 2004). As the I-fronts progress, the remnant cloud is eventually dispersed, with stars of different masses remaining in the same volume. Low- and intermediate-mass young stars in bright-rimmed or comet-shaped clouds on the border of an $\mathrm{OB}$ association are more likely to be formed by triggering. Assuming a shock speed of $\sim 10 \mathrm{~km} \mathrm{~s}^{-1}$, this would result in an age spread of several Myrs between member stars or star groups formed in the sequence. If the velocities of the shocks are higher, as in the case of a supernova explosion, the age spreads would be less.

\section{CONCLUSIONS}

We first selected CTTSs and HAeBe stars in $\lambda$ Ori, Ori OB1, and Lac OB1 based on the 2MASS colors. These PMS stars are then utilized to trace recent star-forming activities. The young stars are found to be lined up in an age sequence between massive stars and comet-shaped clouds or bright-rimmed clouds, with the youngest stars located near the cloud surfaces. There are no PMS stars far behind the I-fronts. These results support the scenario by which the Lyman continuum photons of a luminous $\mathrm{O}$ star create expanding I-fronts that would cause the evaporation and compression of nearby clouds to form BRCs or cometshaped clouds, thereby inducing the birth of low- and intermediate-mass stars. The BRCs provide us with a convenient setting in which to see that the HAeBe stars tend to form in the inner, denser parts of a cloud, whereas the CTTSs are formed near the photoevaporating cloud layers. Young stars in bright-rimmed or comet-shaped clouds near a massive star are likely to have been formed by triggering. Assuming a shock speed of $\sim 10 \mathrm{~km} \mathrm{~s}^{-1}$, this would result in an age spread of several Myrs between member stars or star groups formed in the sequence.

We want to particularly thank Richard F. Green, Director of KPNO, who kindly pro- 
vided us the director's discretionary time to accomplish this work. We are also grateful to the staff of the Beijing Astronomical Observatory for their assistance during our observation runs and to the referee, Hans Zinnecker, for his suggestions helpful in improving the quality of this paper. This research makes use of data products from the Two Micron All Sky Survey, which is a joint project of the University of Massachusetts and the Infrared Processing and Analysis Center/California Institute of Technology, funded by the National Aeronautics and Space Administration and the National Science Foundation (NSF). We also used the Southern H-Alpha Sky Survey Atlas (SHASSA), supported by the NSF. We acknowledge the financial support of grant NSC92-2112-M-008-047 from the National Science Council and 92-N-FA01-1-4-5 from the Ministry of Education of Taiwan.

\section{REFERENCES}

Alcalá, J. M., Wachter, S., Covino, E., Sterzik, M. F., Durisen, R. H., Freyberg, M. J., Hoard, D. W., \& Cooksey, K. 2004, A\&A, 416, 677

Bertoldi, F. 1989, ApJ, 346, 735

Bertoldi, F. \& McKee, C. F. 1990, ApJ, 354, 529

Blaauw, A. 1958, AJ, 63, 186

Blaauw, A. 1961, Bull. Astron. Inst. Netherlands, 15, 265

Blaauw, A. 1964, ARA\&A, 2, 213

Blaauw, A. 1991, in The Physics of Star Formation and Early Stellar Evolution, ed. C. J. Lada \& N. D. Kylafis (Dordresht:Kluwer), 125

Briceño, C., et al. 2006, in Protostars and Planets V, ed. Reipurth, B., Jewitt, D., \& Keil, K. (Tucson: Univ. Arizona Press)

Brown, A. G. A., de Geus, E. J., \& de Zeeuw, P. T. 1994, A\&A, 289, 101

Clark, P. C., Bonnell, I. A., Zinnecker, H., \& Bate, M. R. 2005, MNRAS, 359, 809

Cutri, R. M., et al. 2003, 2MASS All Sky Catalog of Point Sources (Pasadena:IPAC)

Dame, T. M., Hartmann, D., \& Thaddeus, P. 2001, ApJ, 547, 792

Dennison, B., Simonetti, J. H., \& Topasna, G. A. 1998, PASA, 15, 147 
de Bruijne, J. H. J. 1999, MNRAS, 310, 585

de Geus, E. J., de Zeeuw, P. T., \& Lub, J. 1989, A\&A, 216, 44

Deharveng, L., Zavagno, A., \& Caplan, J., 2005, A\&A, 433, 565

de Zeeuw, P. T., Hoogerwerf, R., de Bruijne, J. H. J., Brown, A. G. A., \& Blaauw, A. 1999, AJ, 117,354

Dolan, C. J., \& Mathieu, R. D. 1999, AJ, 118, 2409

Dolan, C. J., \& Mathieu, R. D. 2001, AJ, 121, 2124

Dolan, C. J., \& Mathieu, R. D. 2002, AJ, 123, 387

Duerr, R., Imhoff, C. L., \& Lada, C. J. 1982, ApJ, 261, 135

Dyson, J. E., \& Williams, D. A. 1997, The Physics of the Interstellar Medium (2nd ed.; Bristol: Institute of Physics Publishing)

Elmegreen, B. G. \& Lada, C. J. 1977, ApJ, 214, 725

Elmegreen, B. G. 1998, in ASP Conf. Ser. 148: Origins, ed. C. E. Woodward, J. M. Shull, \& H. A. Thronson, Jr. (San Francisco: ASP), 150

ESA. 1997, The Hipparcos and Tycho Catalogs, 1997, ESA SP-1200

Finkbeiner, D. P. 2003, ApJS, 146, 407

Gaustad, J. E., McCullough, P. R., Rosing, W., \& Van Buren, D. 2001, PASP, 113, 1326

Gies, D. R., \& Bolton, C. T. 1986, ApJS, 61, 419

Haffner, L. M., Reynolds, R. J., Tufte, S. L., Madsen, G. J., Jaehnig, K. P., \& Percival, J. W. 2003, ApJS, 149, 405

Healy, K. R., Hester, J. J., \& Claussen, M. J. 2004, ApJ, 610, 835

Herbig, G. H. 1962, ApJ, 135, 736

Hester, J. J., et al. 1996, AJ, 111, 2349

Hester, J. J., Desch, S. J., Healy, K. R., \& Leshin, L. A. 2004, Science, 304, 1116

Hester, J. J., \& Desch, S. J. 2005, ASP Conf. Ser. 341: Chondrites and the Protoplanetary Disk, 341, 107 
Karr, J. L., \& Martin, P. G. 2003, ApJ, 595, 900

Kenyon, S. J., \& Hartmann, L. 1995, ApJS, 101, 117

Kenyon, S. J., Brown, D. I., Tout, C. A., \& Berlind, P. 1998, AJ, 115, 2491

Kessel-Deynet, O., \& Burkert, A. 2003, MNRAS, 338, 545

Kun, M., Aoyama, H., Yoshikawa, N., Kawamura, A., Yonekura, Y., Onishi, T., \& Fukui, Y. 2001, PASJ, 53, 1063

Kun, M., Prusti, T., Nikolić, S., Johansson, L. E. B., \& Walton, N. A. 2004, A\&A, 418, 89

Lada, C. J. 1987, in IAU Symp. 115, Star Forming Regions, ed. J. Jugaku \& M. Peimbert (Dordrecht: Reidel), 1

Lang, W. J., Masheder, M. R. W., Dame, T. M., \& Thaddeus, P. 2000, A\&A, 357, 1001

Larosa, T. N. 1983, ApJ, 274, 815

Lee, H.-T., Chen, W. P., Zhang, Z. W., \& Hu, J. Y. 2005, ApJ, 624, 808 (Paper I)

Lefloch, B. \& Cernicharo, J. 2000, ApJ, 545, 340

Lefloch, B., Cernicharo, J., Rodríguez, L. F., Miville-Deschênes, M. A., \& Cesarsky, D. 2002, ApJ, 581, 335

Magakian, T. Y. 2003, A\&A, 399, 141

McGroarty, F., Ray, T. P., \& Bally, J. 2004, A\&A, 415, 189

Meyer, M. R., Calvet, N., \& Hillenbrand, L. A. 1997, AJ, 114, 288

O’dell, C. R., Wen, Z., \& Hu, X. 1993, ApJ, 410, 696

Odenwald, S., Fischer, J., Lockman, F. J., \& Stemwedel, S. 1992, ApJ, 397, 174

Ogura, K. \& Sugitani, K. 1998, PASA, 15, 91

Olano, C. A., Walmsley, C. M., \& Wilson, T. L. 1994, A\&A, 290, 235

Preibisch, T. \& Zinnecker, H. 1999, AJ, 117, 2381

Preibisch, T., \& Zinnecker, H. 2006, astro-ph/0610826 
Sanchawala, K., Chen, W. P., Lee, H. T., Nakajima, Y., Tamura, H., Daisuke, B., Sato, S., \& Chu, Y. H., 2006, ApJ, in submission, astro-ph0603043

Schaerer, D. \& de Koter, A. 1997, A\&A, 322, 598

Schlegel, D. J., Finkbeiner, D. P., \& Davis, M. 1998, ApJ, 500, 525

Spitzer, L. 1998, Physical Processes in the Interstellar Medium (New York: WileyInterscience)

Stanke, T., Smith, M. D., Gredel, R., \& Szokoly, G. 2002, A\&A, 393, 251

Sugitani, K., Fukui, Y., \& Ogura, K. 1991, ApJS, 77, 59

Sugitani, K., \& Ogura, K. 1994, ApJS, 92, 163

Whitworth, A. P., \& Zinnecker, H. 2004, A\&A, 427, 299

White, G. J., Casali, M. M., \& Eiroa, C. 1995, A\&A, 298, 594

White, R. J., \& Hillenbrand, L. A. 2005, ApJ, 621, L65

Zacharias, N., Urban, S. E., Zacharias, M. I., Wycoff, G. L., Hall, D. M., Monet, D. G., \& Rafferty, T. J. 2004, AJ, 127, 3043

Zavagno, A., Deharveng, L., Comerón, F., Brand, J., Massi, F., Caplan, J., \& Russeil, D., A\&A, 446, 171 
Table 1. REGIONS STUDIED

\begin{tabular}{ccc}
\hline \hline Region & \multicolumn{2}{c}{ Approximate Coordinates } \\
\hline Lac OB1 $^{a}$ & $\ell \sim 83^{\circ}$ to $112^{\circ}$ & $b \sim-3.5$ to $-25^{\circ} .7$ \\
Trapezium $^{b}$ & RA $\sim 5^{h} 03^{m}$ to $5^{h} 32^{m}$ & DEC $\sim-1^{\circ} 45^{\prime}$ to $-8^{\circ} 10^{\prime}$ \\
$\lambda$ Ori $^{c}$ & RA $\sim 5^{h} 23^{m}$ to $5^{h} 52^{m}$ & DEC $\sim+6^{\circ} 40^{\prime}$ to $+14^{\circ} 22^{\prime}$ \\
Ori East & RA $\sim 5^{h} 52^{m}$ to $5^{h} 57^{m}$ & DEC $\sim+1^{\circ} 15^{\prime}$ to $+2^{\circ} 15^{\prime}$ \\
Control Field 1 & $\ell \sim 192^{\circ}$ to $260^{\circ}$ & $b \sim+15^{\circ}$ to $+44^{\circ}$ \\
Control Field 2 & RA $\sim 20^{h} 24^{m}$ to $21^{h} 05^{m}$ & DEC $\sim+25^{\circ} 16^{\prime}$ to $+32^{\circ} 55^{\prime}$ \\
\hline
\end{tabular}

ancluding BRC LBN 437 and comet-shaped cloud GAL 110-13

b'Including BRCs, IC 2118, LDN 1616 and LDN 1634

'Including BRCs, B 30 and B 35

Table 2. Imaging Observations

\begin{tabular}{ccccc}
\hline \hline Fields & $\begin{array}{c}\text { RA } \\
(\mathrm{J} 2000)\end{array}$ & $\begin{array}{c}\text { DEC } \\
(\mathrm{J} 2000)\end{array}$ & Filter & $\begin{array}{c}\text { Total Exp. Time } \\
(\mathrm{s})\end{array}$ \\
\hline B30 & $05: 29: 51.4$ & $+12: 13: 58$ & $\mathrm{H} \alpha$ & 5400 \\
B35 & $05: 44: 20.0$ & $+09: 10: 40$ & $\mathrm{H} \alpha$ & 5400 \\
Ori East & $05: 53: 58.6$ & $+01: 40: 37$ & $\mathrm{H} \alpha$ & 3600 \\
LDN 1616 & $05: 07: 06.0$ & $-03: 17: 54$ & $\mathrm{H} \alpha$ & 7200 \\
LDN 1634 & $05: 20: 16.0$ & $-05: 49: 28$ & $\mathrm{H} \alpha$ & 3600 \\
IC 2118 & $05: 07: 44.0$ & $-06: 12: 35$ & $\mathrm{H} \alpha$ & 2400 \\
LBN 437 & $22: 34: 31.0$ & $+40: 37: 44$ & $\mathrm{H} \alpha$ & 3600 \\
LBN 437 & $22: 34: 31.0$ & $+40: 37: 44$ & {$[\mathrm{~S} \mathrm{II}]$} & 7200 \\
\hline
\end{tabular}


Table 3. CTTS and CTTS Candidates

\begin{tabular}{|c|c|c|c|c|c|}
\hline $\operatorname{Star}^{a}$ & 2MASS & Emission Line $(\mathrm{s})^{b}$ & $\mathrm{Li}^{c}$ & Obs. $^{d}$ & Remarks \\
\hline 1 & J05065464-0320047 & $\mathrm{H}(-50.6), \mathrm{O}(-0.6), \mathrm{Ca}, \mathrm{He}$ & $\mathrm{a}$ & $\mathrm{K}$ & LkHa 333, associated with LDN 1616 \\
\hline 2 & J05073016-0610158 & $\mathrm{H}(-92.5), \mathrm{O}(-10.8), \mathrm{S}(-1.4), \mathrm{Fe}, \mathrm{Ca}, \mathrm{He}$ & $\mathrm{a}$ & $\mathrm{K}$ & associated with IC 2118 \\
\hline 3 & J05073060-0610597 & $\mathrm{H}(-23.3), \mathrm{Ca} ?$ & $\mathrm{a}$ & $\mathrm{K}$ & associated with IC 2118 \\
\hline 4 & J05122053-0255523 & $\mathrm{H}(-12.1)$ & $\mathrm{a}$ & $\mathrm{K}$ & V531 Ori \\
\hline 5 & J05141328-0256411 & $\mathrm{H}(-210.6), \mathrm{O}(-2.5), \mathrm{Fe}, \mathrm{Ca}, \mathrm{He}$ & $\mathrm{n}$ & $\mathrm{K}$ & Kiso A-0975 16 \\
\hline 6 & J05152683-0632010 & $\mathrm{H}(-0.3)$ & $\mathrm{a}$ & $\mathrm{K}$ & $\mathrm{H} \alpha$ emission is week,could be a WTTS \\
\hline 7 & J05162251-0756503 & $\mathrm{H}(-37.7), \mathrm{O}(-1.0), \mathrm{Ca}, \mathrm{He}$ & $\mathrm{a}$ & $\mathrm{K}$ & \\
\hline 8 & J05181685-0537300 & $\mathrm{H}(-57.3), \mathrm{O}(-2.3), \mathrm{Fe}, \mathrm{Ca}, \mathrm{He}$ & $\mathrm{n}$ & $\mathrm{K}$ & Kiso A-0975 43 \\
\hline 9 & J05191356-0324126 & $\mathrm{H}(-52.1), \mathrm{Ca}, \mathrm{He}$ & $\mathrm{a}$ & $\mathrm{K}$ & Kiso A-0975 45 \\
\hline 10 & J05191549-0204529 & $\mathrm{H}(-10.7), \mathrm{O}(-3.6), \mathrm{Ca}$ & a & $\mathrm{K}$ & \\
\hline 11 & J05201945-0545553 & $\mathrm{H}(-26.9), \mathrm{Ca}, \mathrm{He}$ & $\mathrm{a}$ & $\mathrm{K}$ & Kiso A-0975 52, IRAS 05178-0548, associated with LDN 1634 \\
\hline 12 & J05202573-0547063 & $\mathrm{H}(-100.2), \mathrm{O} ?, \mathrm{Fe}, \mathrm{Ca}, \mathrm{He}$ & $\mathrm{a}$ & $\mathrm{K}$ & V534 Ori, associated with LDN 1634 \\
\hline 13 & J05203142-0548247 & $\mathrm{H}(-19.3), \mathrm{Ca}, \mathrm{He}$ & a & $\mathrm{K}$ & StHA 39, associated with LDN 1634 \\
\hline 14 & J05253979-0411020 & $\mathrm{H}(-138.9), \mathrm{Fe}, \mathrm{Ca}, \mathrm{He}$ & $\mathrm{n}$ & $\mathrm{K}$ & Kiso A-0975 86 \\
\hline 15 & J05262158+1131339 & $\mathrm{H}(-15.4), \mathrm{O}(-1.8), \mathrm{Fe}, \mathrm{Ca}$ & - & $\mathrm{B}$ & IRAS $05235+1129$ \\
\hline 16 & J05292393+1151576 & $\mathrm{H}(-40.5), \mathrm{Ca}, \mathrm{He}$ & $\mathrm{a}$ & $\mathrm{B}, \mathrm{K}$ & V649 Ori, associated with B 30 \\
\hline 17 & J05300203+1213357 & $\mathrm{H}(-33.6), \mathrm{Ca}, \mathrm{He}$ & $\mathrm{a}$ & $\mathrm{B}, \mathrm{K}$ & GX Ori, IRAS $05272+1211$, associated with B 30 \\
\hline 18 & J05301313+1208458 & $\mathrm{H}(-5.7), \mathrm{Ca}$ & $\mathrm{a}$ & $\mathrm{B}, \mathrm{K}$ & GY Ori, associated with B 30 \\
\hline 19 & J05311615+1125312 & $\mathrm{H}(-21.9)$ & $\mathrm{n}$ & $\mathrm{B}, \mathrm{K}$ & V449 Ori \\
\hline 20 & J05315128+1216208 & $\mathrm{H}(-127.3), \mathrm{O}(-10.2), \mathrm{He}$ & $\mathrm{a}$ & $\mathrm{B}, \mathrm{K}$ & associated with B 30 \\
\hline 21 & J05323207+1044178 & $\mathrm{H}(-96.7), \mathrm{Ca}, \mathrm{He}$ & - & B & \\
\hline 22 & J05324305+1221083 & $\mathrm{H}(-13.9), \mathrm{O}(-1.3), \mathrm{Ca}, \mathrm{He}$ & $\mathrm{a}$ & $\mathrm{B}, \mathrm{K}$ & V460 Ori, IRAS 05299+1219, associated with B 30 \\
\hline 23 & J05330207+1137114 & H(-176.8), Fe, Ca, He & - & $\mathrm{B}$ & \\
\hline 24 & J05391268+0915522 & $\mathrm{H}(-215.6), \mathrm{Ca}, \mathrm{He}$ & - & B & \\
\hline 25 & J05432091+0906071 & $\mathrm{H}(-21.8), \mathrm{O}(-0.5), \mathrm{Ca}, \mathrm{He}$ & $\mathrm{a}$ & $\mathrm{B}, \mathrm{K}$ & V625 Ori, IRAS 05406+0904, associated with B 35 \\
\hline 26 & J05440899+0909147 & $\mathrm{H}(-44.5), \mathrm{O}(-2.1), \mathrm{Fe}, \mathrm{Ca}, \mathrm{He}$ & $\mathrm{a}$ & $\mathrm{B}, \mathrm{K}$ & QR Ori, IRAS 05413+0907, associated with B 35 \\
\hline 27 & J05451493+0721223 & $\mathrm{H}(-5.6)$ & $\mathrm{n}$ & $\mathrm{B}, \mathrm{K}$ & V661 Ori \\
\hline
\end{tabular}


Table 3-Continued

\begin{tabular}{cccccl}
\hline \hline \multirow{2}{*}{$\operatorname{Star}^{a}$} & \multirow{2}{*}{ 2MASS } & Emission Line $(\mathrm{s})^{b}$ & $\mathrm{Li}^{c}$ & Obs. $^{d}$ & \\
& & & & \\
\hline 28 & $\mathrm{~J} 05452235+0904123$ & & - & $\mathrm{B}$ & FU Ori, IRAS 05426+0903, associated with B 35 \\
29 & $\mathrm{~J} 05515035+0821066$ & $\mathrm{H}(-181.9), \mathrm{Ca}$ & $\mathrm{a}$ & $\mathrm{B}, \mathrm{K}$ & \\
30 & $\mathrm{~J} 05534090+0138140$ & $\mathrm{H}(-29.0), \mathrm{O}(-2.7), \mathrm{He}$ & - & $\mathrm{B}$ & LkHA 334, IRAS F05510+0137, associated with Ori East \\
31 & $\mathrm{~J} 05535869+0144094$ & $\mathrm{H}(-37.0), \mathrm{Ca}, \mathrm{He}$ & - & $\mathrm{B}$ & LkHA 335, IRAS F05513+0143, associated with Ori East \\
32 & $\mathrm{~J} 21370366+4321172$ & $\mathrm{H}(-174.8), \mathrm{Fe}, \mathrm{Ca}, \mathrm{He}$ & $\mathrm{a}$ & $\mathrm{B}, \mathrm{K}$ & V1082 Cyg \\
33 & $\mathrm{~J} 21395545+4313082$ & $\mathrm{H}(-83.6), \mathrm{Ca}$ & - & $\mathrm{B}$ & \\
34 & $\mathrm{~J} 21535750+4659443$ & $\mathrm{H}(-51.7)$ & - & $\mathrm{B}$ & LkHA 256 \\
35 & $\mathrm{~J} 22361978+4006273$ & $\mathrm{H}(-63.9), \mathrm{O}(-1.8), \mathrm{Ca}$ & - & $\mathrm{B}$ & associated with IRAS 22343+3944 group \\
36 & $\mathrm{~J} 22362779+3954066$ & $\mathrm{H}(-18.4)$ & - & $\mathrm{B}$ & associated with IRAS 22343+3944 group \\
37 & $\mathrm{~J} 22370328+4005185$ & $\mathrm{H}(-10.3), \mathrm{Ca} ?, \mathrm{He} ?$ & $\mathrm{a}$ & $\mathrm{B}, \mathrm{K}$ & associated with IRAS 22343+3944 group \\
38 & $\mathrm{~J} 22371683+3952260$ & $\mathrm{H}(-130.8), \mathrm{O}(-4.5), \mathrm{Ca}$ & - & $\mathrm{B}$ & associated with IRAS 22343+3944 group \\
39 & $\mathrm{~J} 23104483+4508511$ & $\mathrm{H}(-7.9)$ & $\mathrm{n}$ & $\mathrm{B}, \mathrm{K}$ & \\
40 & $\mathrm{~J} 23373847+4824119$ & $\mathrm{H}(-19.2)$ & $\mathrm{a}$ & $\mathrm{B}, \mathrm{K}$ & BM And, associated with GAL 110-13 \\
\hline
\end{tabular}

a Star 1-31 and 32-40 are in the Orion and Lacerta regions, respectively.

${ }^{\mathrm{b}} \mathrm{H}-\mathrm{H} \alpha$, Ca-Ca II (K, H $(3934,3968 \AA)$, and/or infrared triplet $\left.(8498,8542,8662 \AA)\right)$, He-He I $(5876 \AA), \mathrm{O}-[\mathrm{O} \mathrm{I}](6300 \AA)$, S-[S II] $(6717 \AA)$, Fe-Fe II (4924 $\AA$ ), The number following H, O, S, are the equivalent widths of H $\alpha$, [O I], and [S II], respectively.

${ }^{\mathrm{c}} \mathrm{a}-$-absorbtion, $\mathrm{n}-\mathrm{no}$ absorbtion, - - low spectral resolution in $\mathrm{BAO}$

${ }^{\mathrm{d}} \mathrm{B}-\mathrm{BAO}, \mathrm{K}-\mathrm{KPNO}$ 
Table 4. Herbig Ae/Be Stars

\begin{tabular}{|c|c|c|c|c|c|}
\hline $\operatorname{Star}^{a}$ & 2MASS & Emission Line(s) & Sp. Type ${ }^{b}$ & Obs. ${ }^{c}$ & Remarks \\
\hline 41 & J05042998-0347142 & $\mathrm{H}$ & $\mathrm{A} 3 \mathrm{e}$ & K & UX Ori, IRAS 05020-0351, associated with LDN 1616 \\
\hline 42 & J05113654-0222484 & $\mathrm{H}$ & $\mathrm{A} 3 \mathrm{e}$ & $\mathrm{K}$ & \\
\hline 43 & J05305472+1421524 & $\mathrm{H}$ & $\mathrm{F} 2 \mathrm{e}$ & $\mathrm{K}$ & \\
\hline 44 & J05312805+1209102 & $\mathrm{H}, \mathrm{O}$ & $\mathrm{A} 2 \mathrm{e}$ & $\mathrm{K}$ & HK Ori, IRAS 05286+1207, associated with B 30 \\
\hline 45 & J05313515+0951553 & $\mathrm{H}$ & B9e & $\mathrm{K}$ & IRAS $05288+0949$ \\
\hline 46 & J05315724+1117414 & $\mathrm{H}$ & $\mathrm{A} 0 \mathrm{e}$ & $\mathrm{B}$ & HD 244604, IRAS $05291+1115$ \\
\hline 47 & J05350960+1001515 & $\mathrm{H}, \mathrm{O} ?$ & B9e & B & V1271 Ori, IRAS $05324+0959$ \\
\hline 48 & J05390921+0925301 & $\mathrm{H}$ & F7e & $\mathrm{B}, \mathrm{K}$ & V506 Ori \\
\hline 49 & $\mathrm{~J} 21462666+4744154$ & $\mathrm{H}, \mathrm{O}$ & B9e & K & \\
\hline 50 & $\mathrm{~J} 21514726+4615115$ & $\mathrm{H}$ & A9e & $\mathrm{K}$ & LR Cyg \\
\hline 51 & $\mathrm{~J} 22154039+5215559$ & $\mathrm{H}$ & $\mathrm{A} 2 \mathrm{e}$ & $\mathrm{B}$ & \\
\hline 52 & $\mathrm{~J} 22344101+4040045$ & $\mathrm{H}, \mathrm{O}, \mathrm{S}$ & $\mathrm{A} 2 \mathrm{e}$ & $\mathrm{K}$ & V375 Lac \\
\hline 53 & $\mathrm{~J} 22363511+4000156$ & $\mathrm{H}, \mathrm{O}$ & $\mathrm{B} 8 \mathrm{e}$ & $\mathrm{B}$ & associated with IRAS $22343+3944$ group \\
\hline
\end{tabular}

${ }^{a}$ Star 41-48 and 49-53 are in the Orion and Lacerta regions, respectively.

${ }^{\mathrm{b}} \mathrm{H}-\mathrm{H} \alpha, \mathrm{O}-[\mathrm{O} \mathrm{I}](6300 \AA), \mathrm{S}-[\mathrm{S}$ II] $(6717 \AA)$

${ }^{\mathrm{c}} \mathrm{B}-\mathrm{BAO}, \mathrm{K}-\mathrm{KPNO}$ 
Table 5. Non-PMS Stars

\begin{tabular}{lcccl}
\hline \hline Star & 2 MASS & Sp. Type & Obs. $^{a}$ & \\
& & & & \multicolumn{1}{c}{ Remarks } \\
\hline 54 & J05232026+0934432 & A0 & B, K & TYC 704-1857-1 \\
55 & J05285405-0606063 & Me & K & Kiso A-0975 119, IRAS 05264-0608 \\
56 & J05413010+1418225 & C & K & BC 203 \\
57 & J05442880+0652019 & M & B & \\
58 & J05464207+0643469 & C & B & IRAS 05440+0642 \\
59 & J05480851+0954012 & Ce & B, K & V638 Ori, IRAS 05453+0953 \\
60 & J07323273+2647156 & C & K & objectFBS 0729+269 \\
61 & J07475919+2052254 & Ce & K & \\
62 & J08231037-0153257 & C & K & \\
63 & J08292902+1046241 & C & K & FBS 0826+109 \\
64 & J08423302+0621195 & M & K & \\
65 & J08541870-1200541 & Ce & K & IRAS 08519-1149 \\
66 & J09111450-0922053 & Me & K & VV Hya \\
67 & J0933061-2216282 & M & K & \\
68 & J20245404+2609115 & M & B & \\
69 & J20291739+2617284 & Me & B & IRAS 20271+2607 \\
70 & J20304177+2812340 & M & B & DU Vul, IRAS 20285+2802 \\
71 & J20311267+2612270 & M & B & \\
72 & J20415136+2752525 & M & B & IRAS 20397+2742 \\
73 & J20532040+2516196 & C & B & \\
74 & J20551307+3254065 & M & B & \\
75 & J20555284+2640515 & M & B & UY Vul, IRAS 20537+2629 \\
76 & J21040556+2632111 & M & B & V444 Vul, IRAS 21019+2620 \\
77 & J21244172+4437134 & Ce & B & V1563 Cyg, IRAS 21228+4424 \\
78 & J21383182+4542469 & Ce & K & V1568 Cyg, IRAS 21366+4529 \\
79 & J21595030+3313596 & M & B & \\
80 & J22024329+4216400 & BL Lac & K & BL Lac \\
& & & &
\end{tabular}


Table 5-Continued

\begin{tabular}{lcccl}
\hline \hline Star & \multirow{2}{*}{ 2MASS } & Sp. Type & Obs. $^{a}$ & \multicolumn{1}{c}{ Remarks } \\
& & & & \\
\hline 81 & J22055958+3530057 & M & B & XX Peg \\
82 & J22070988+2828374 & M & B & V392 Peg, IRAS F22048+2813 \\
83 & J22075421+4105113 & M & B & V379 Lac, IRAS 22057+4050 \\
84 & J22084406+4855248 & M & B & V426 Lac, IRAS 22067+4840 \\
85 & J22121336+4646065 & C & B & IRAS 22101+4631 \\
86 & J22135091+2447203 & M & B & \\
87 & J22213857+3335586 & C & B & \\
88 & J22261658+4221089 & A0 & B & \\
89 & J22295650+4546539 & Ce & B & V386 Lac \\
90 & J22313443+4816005 & C & K & V387 Lac, IRAS 22294+4800 \\
91 & J22314368+4748038 & PN & K & PN G100.0-08.7, IRAS 22296+4732 \\
92 & J22451504+5051534 & Ce & B & HL Lac, IRAS 22431+5036 \\
93 & J22491976+5154487 & M & B & IRAS 22472+5138 \\
94 & J22514566+4921137 & C & B & IRAS 22495+4905 \\
95 & J22521809+3413364 & M & B & IRAS 22499+3357 \\
96 & J22592372+4811589 & Me & K & \\
97 & J23023314+4649483 & M & B & NSV 14395, IRAS 23002+4633 \\
98 & J23113005+4702525 & M & B & IRAS 23092+4646 \\
99 & J23175960+4645122 & M & B & AO And, IRAS 23156+4628 \\
\hline
\end{tabular}

${ }^{\mathrm{a}} \mathrm{B}-\mathrm{BAO}, \mathrm{K}-\mathrm{KPNO}$ 
Table 6. Differences between triggered and spontaneous star formation

\begin{tabular}{lll}
\hline \hline & \multicolumn{1}{c}{ Triggered } & \multicolumn{1}{c}{ Spontaneous } \\
\hline Sequential star formation & $\begin{array}{l}\text { Yes. PMS stars close to triggering } \\
\text { sources are older than those close to BRCs }\end{array}$ & No \\
Stellar distribution & $\begin{array}{l}\text { PMS stars are located between triggering } \\
\text { sources or around surfaces of BRCs with no } \\
\text { Star formation efficiency }\end{array}$ & PMS stars can be anywhere, including being far \\
& High & behind the surface of a BRC \\
& & Low, less than a few percent. \\
\hline
\end{tabular}


Table 7. Proper Motions of Stars in GAL 110-13 and Lac OB1 Subgroups a and b

\begin{tabular}{ccccccc}
\hline \hline Star & Sp. type & $\begin{array}{c}\text { pmRA } \\
(\mathrm{mas} / \mathrm{yr})\end{array}$ & $\begin{array}{c}\text { pmDEC } \\
(\mathrm{mas} / \mathrm{yr})\end{array}$ & $\begin{array}{c}\text { e_pmRA } \\
(\mathrm{mas} / \mathrm{yr})\end{array}$ & $\begin{array}{c}\text { e_pmDEC } \\
(\mathrm{mas} / \mathrm{yr})\end{array}$ & Reference \\
\hline HD 222142 & B9.5 V & 0.3 & -3.1 & 0.6 & 0.6 & UCAC2 \\
HD 222086 & B9 V & 0.5 & -2.8 & 1.0 & 1.1 & UCAC2 \\
HD 222046 & B8 Vp & 0.4 & -2.7 & 1.0 & 1.0 & UCAC2 \\
Star 40 & Continuum & 3.4 & -7.8 & 2.7 & 2.6 & UCAC2 \\
Lac OB1a & - & -0.3 & -3.7 & - & - & Hipparcos \\
Lac OB1b & - & -0.5 & -4.6 & - & - & Hipparcos \\
\hline
\end{tabular}



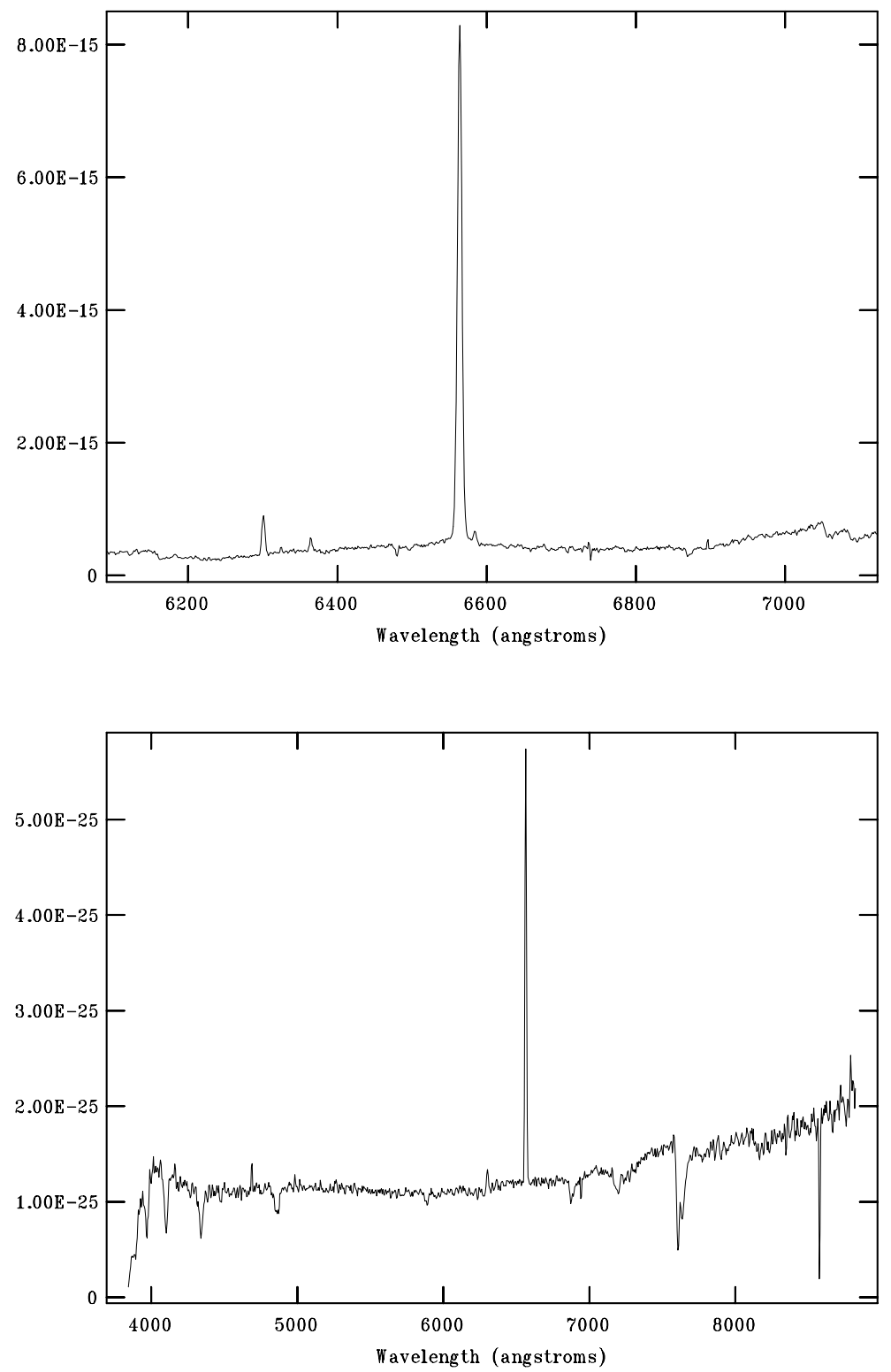

Fig. 1. - Example spectra (top) for stars 20 (bottom) and 53. Star 20 is a CTTS and shows a veiled continuum with strong $\mathrm{H} \alpha$ and [O I], 6300 and $6363 \AA$ emission lines. Star 53 is an HAeBe star and shows the $\mathrm{H} \alpha$ in emission but the other Balmer lines in absorption. 


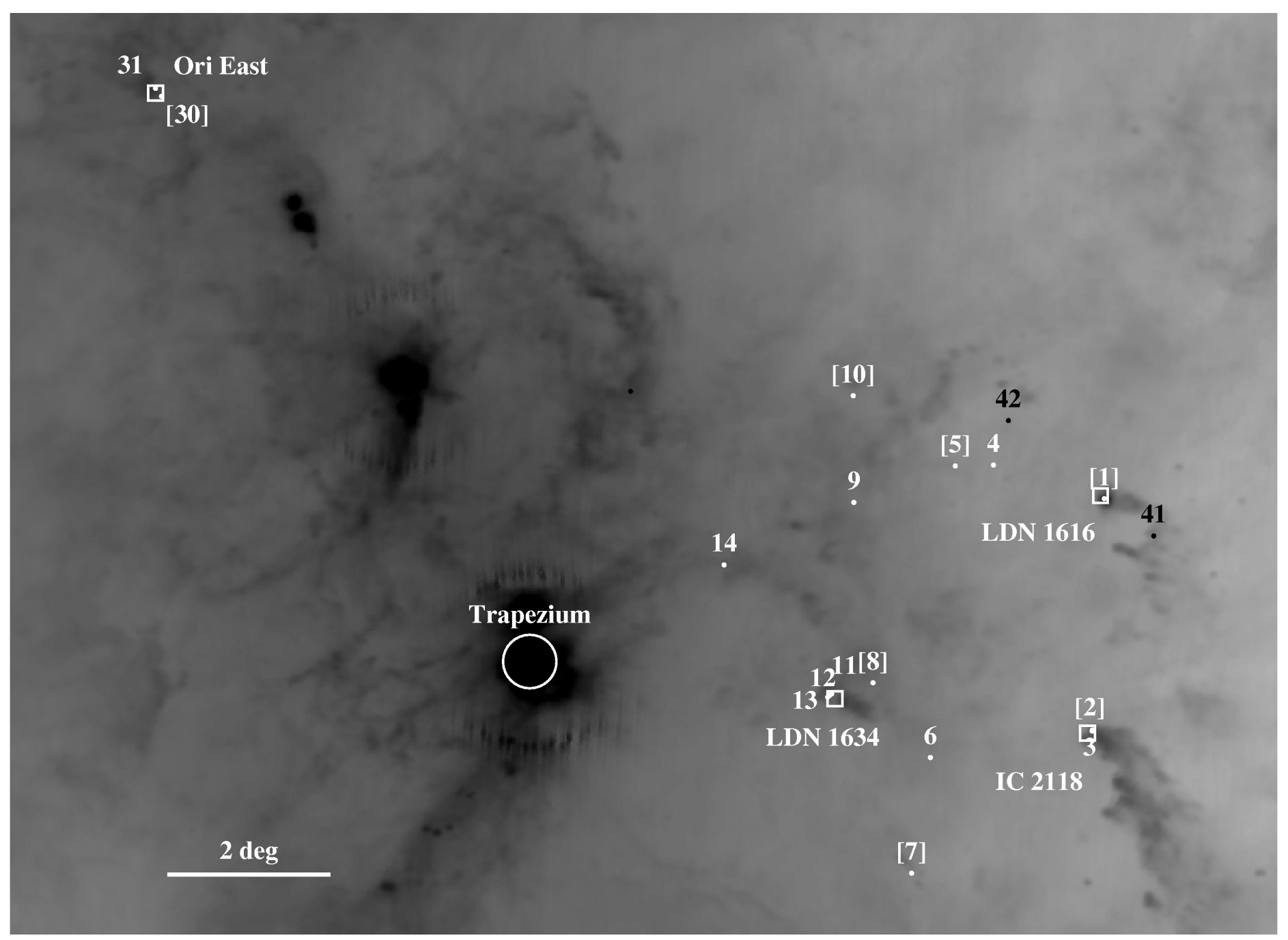

Fig. 2.- IRAS $100 \mu \mathrm{m}$ image of the Trapezium region. The dots indicate CTTSs (white) and HAeBe stars (black), labeled with the identification numbers from Tables 3 and 4. PMS stars with forbidden line(s) are bracketed. The boxes mark the fields of the $\mathrm{H} \alpha$ images shown in (Fig. 4). East is to the left and north to the top. 


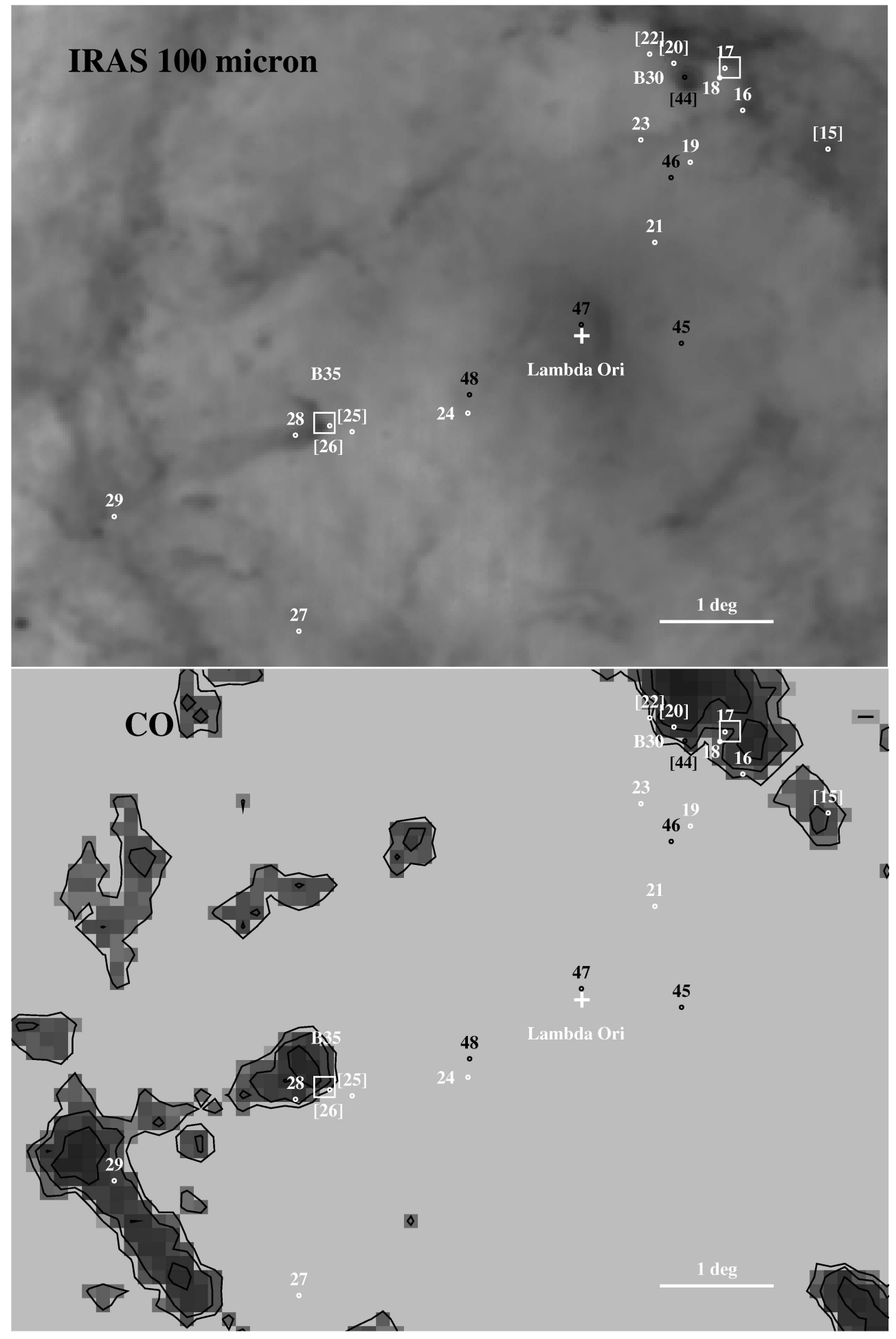

Fig. 3.- IRAS $100 \mu \mathrm{m}$ and CO images of the $\lambda$ Ori region. The symbols are the same as in Figure 2. The distribution of PMS stars extends from $\lambda$ Ori to B 30 and B 35. East is to the left and north to the top. 


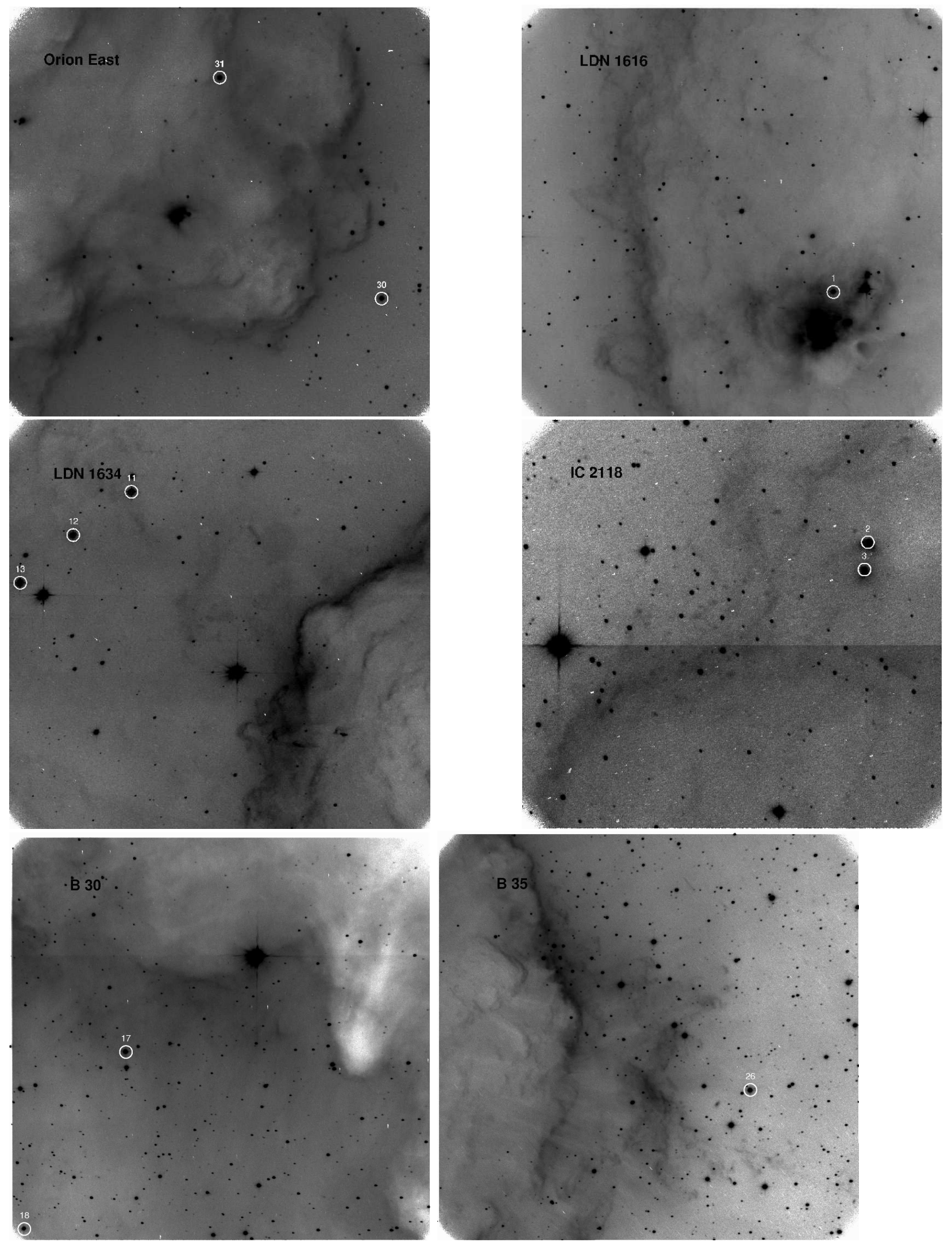

Fig. 4.- $\mathrm{H} \alpha$ images of the Ori OB1 BRCs. The stars in Table 3 are marked. East is to the left and north to the top. The field of view of each image is $\sim 11^{\prime}$. 

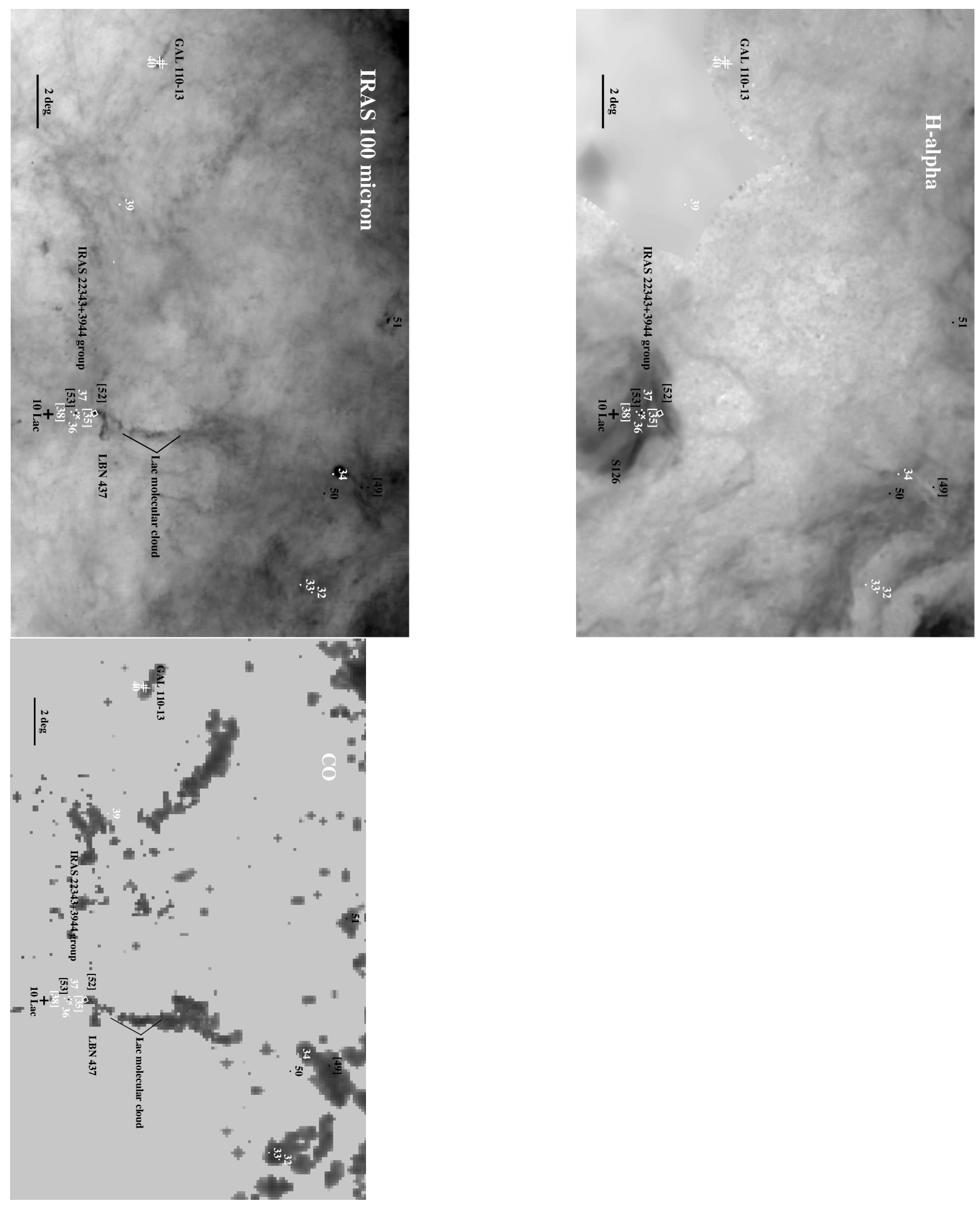

Fig. 5.- IRAS $100 \mu \mathrm{m}, \mathrm{H} \alpha$ and $\mathrm{CO}$ images of the Lac OB1 association using the orientation of the Galactic coordinates, i.e., north is to the top and the Galactic longitude increases to the left. The white plus signs indicate the 3 late-B stars, HD 222142, HD 222086, and HD 222046, in GAL 110-13 and the cross marks indicate the CTTS candidate in the IRAS $22343+3944$ group, 2MASS J22354224+3959566. The other symbols are the same as in Fig. 2, 


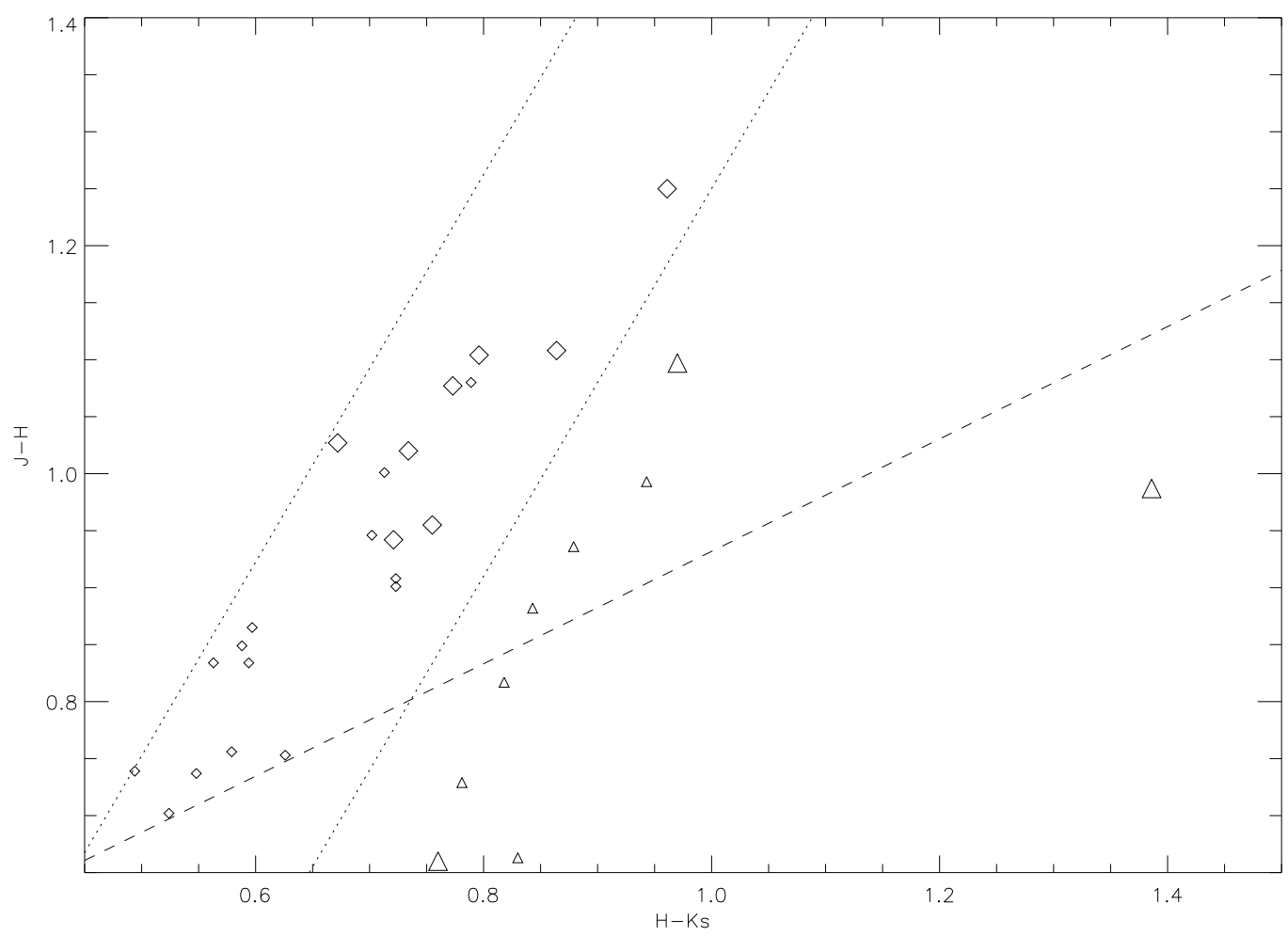

Fig. 6.- 2MASS color-color diagram of the CTTSs (diamonds) and HAeBe stars (triangles) from Tables 3 and 4. Here we only present the CTTSs with a Li absorption line detected in their spectra. CTTSs and HAeBe stars with forbidden lines are indicated by larger symbols. PMS stars with forbidden line are redder than those without. The dotted and dashed lines represent the reddening direction and the dereddened CTTS locus, respectively. CTTSs and HAeBe stars are well separated by the line $\left(j \_m-h \_m\right)-1.7\left(h \_m-k \_m\right)+0.450=0$. 

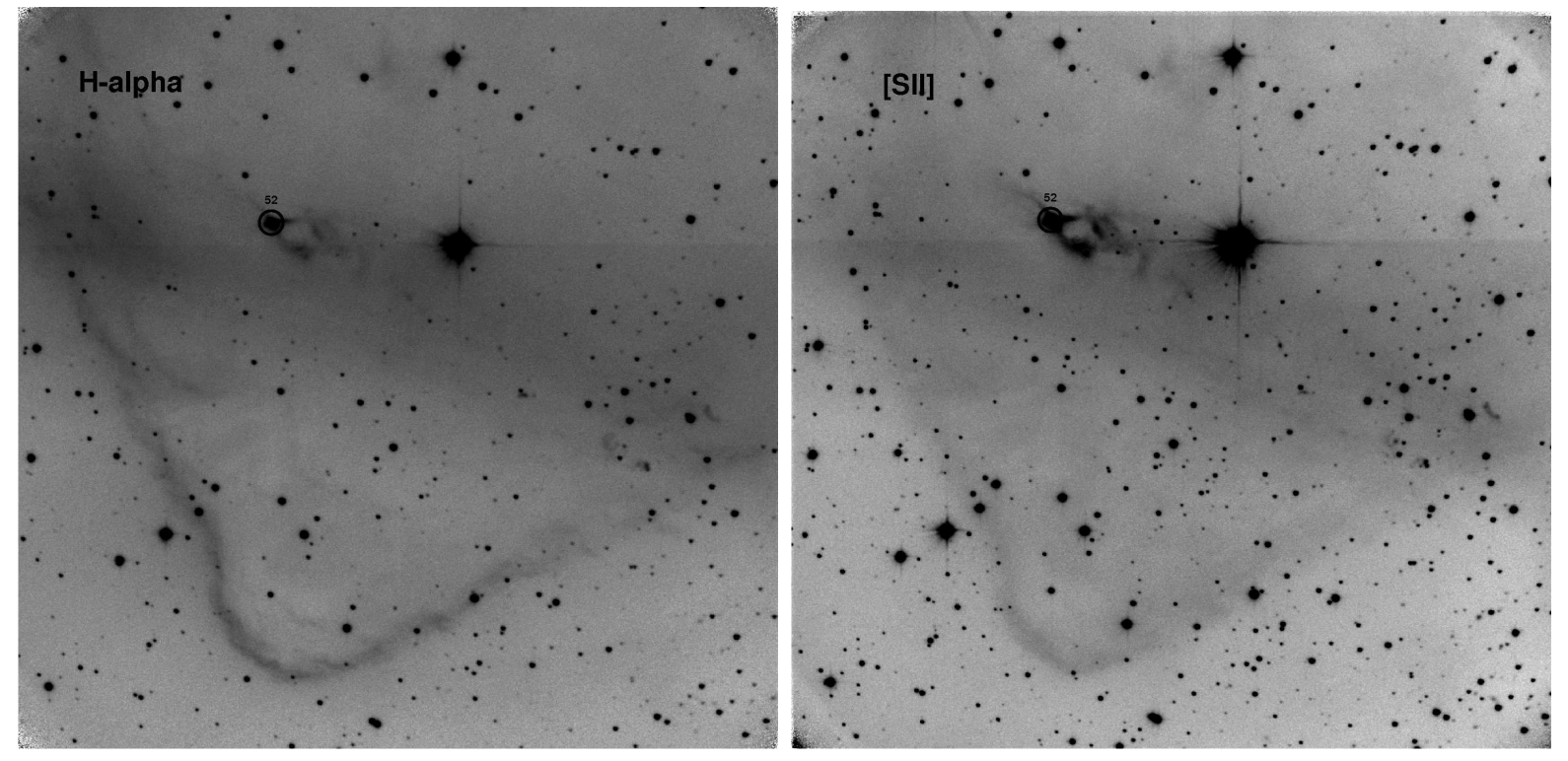

Fig. 7.- $\mathrm{H} \alpha$ and [S II] images of LBN 437. Star 52 is associated with the nebulosity HH 398. East is to the left and north to the top. The field of view of each image is $\sim 11^{\prime}$ 


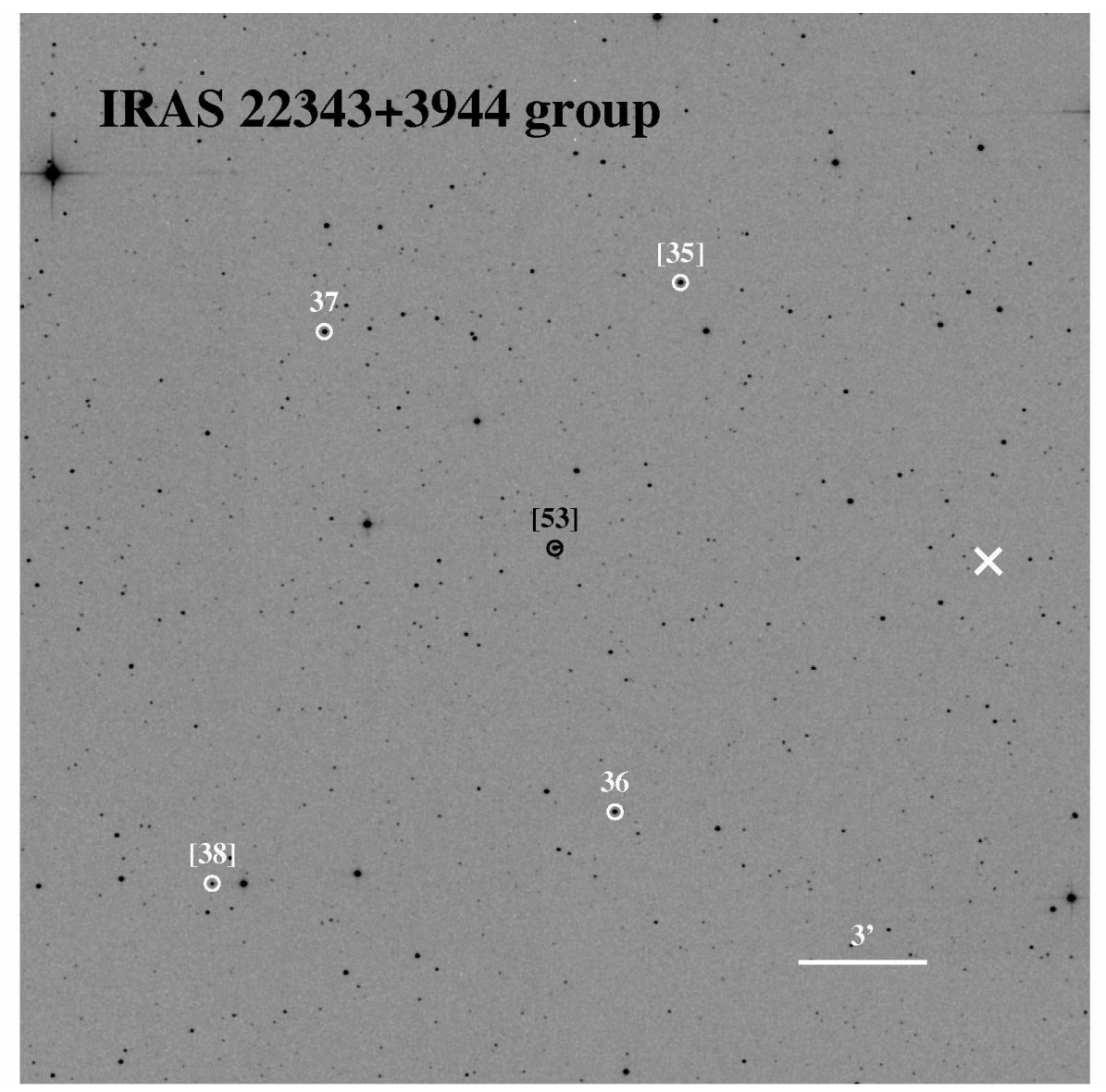

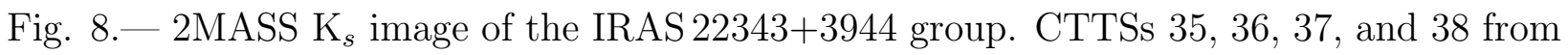
Table 3 (in white) and HAeBe star 53 (in black) are labeled. The cross indicates the CTTS candidate, 2MASS J22354224+3959566. The other symbols are the same as in Fig. 2, East is to the left and north to the top. 


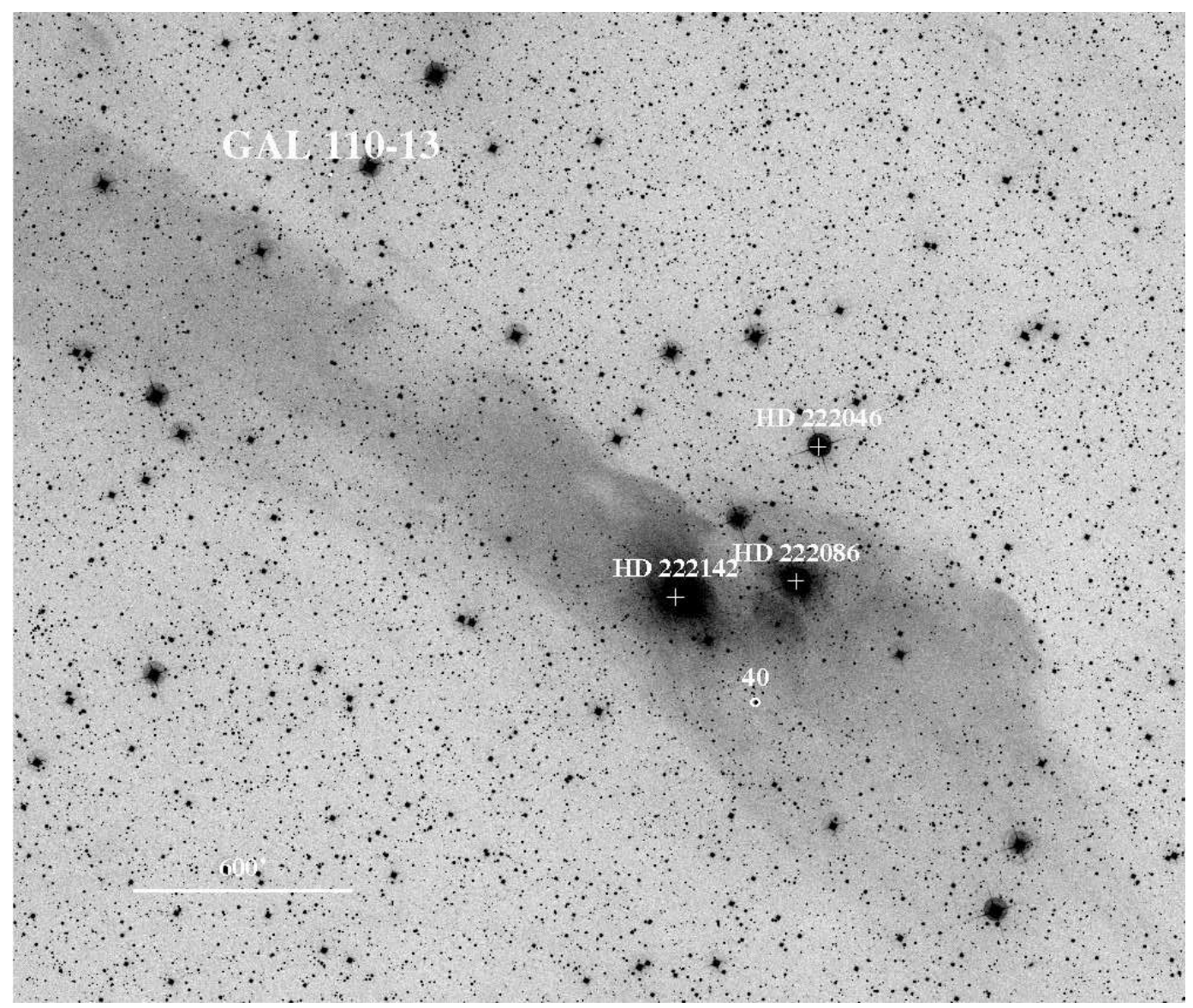

Fig. 9.- DSS blue image of the comet-shaped cloud GAL 110-13. Star 40 (CTTS) and three late-B stars are marked. The Galactic longitude and latitude increase to the left and to the top, respectively. 

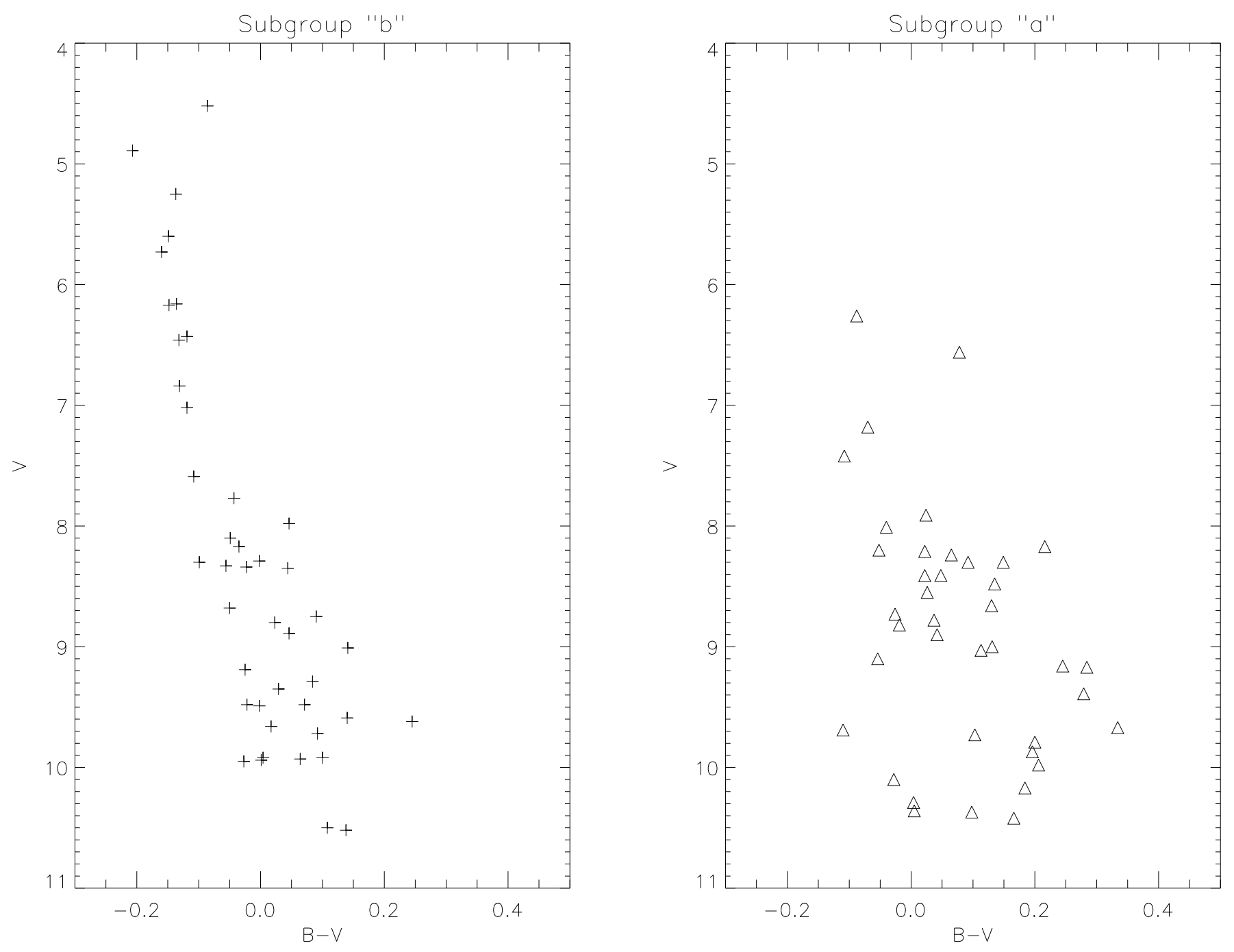

Fig. 10.- Color-magnitude diagrams of the subgroups Lac OB1a and Lac OB1b. The stars in Lac OB1b (pluses) form a clear main sequence, whereas those in Lac OB1a (triangles) are scattered to the right of the sequence, implying a younger age for Lac OB1a. 